\title{
High-Resolution, Rapid-Scan Dual-Doppler Retrievals of Vertical Velocity in a Simulated Supercell
}

\author{
Nathan A. Dahl, ${ }^{\mathrm{a}, \mathrm{b}}$ Alan Shapiro, ${ }^{\mathrm{c}, \mathrm{d}}$ Corey K. Potvin,,${ }^{\mathrm{a}, \mathrm{c}, \mathrm{e}}$ AdAm Theisen, ${ }^{\mathrm{f}}$ Joshua G. Gebauer, $^{\mathrm{c}}$ \\ AleXANDER D. SCHENKMAN, ${ }^{\mathrm{g}}$ AND Ming XUE ${ }^{\mathrm{d}, \mathrm{c}}$ \\ ${ }^{\text {a }}$ Cooperative Institute for Mesoscale Meteorological Studies, University of Oklahoma, Norman, Oklahoma \\ ${ }^{\mathrm{b}}$ NOAA/National Weather Service/Storm Prediction Center, Norman, Oklahoma \\ ${ }^{\mathrm{c}}$ School of Meteorology, University of Oklahoma, Norman, Oklahoma \\ ${ }^{\mathrm{d}}$ Center for Analysis and Prediction of Storms, University of Oklahoma, Norman, Oklahoma \\ ${ }^{\mathrm{e}}$ NOAA/National Severe Storms Laboratory, Norman, Oklahoma \\ ${ }^{\mathrm{f}}$ Argonne National Laboratory, Lemont, Illinois \\ ${ }^{\mathrm{g}}$ Berkshire Hathaway Specialty Insurance, Boston, Massachusetts
}

(Manuscript received 27 November 2018, in final form 9 May 2019)

\begin{abstract}
Observation system simulation experiments are used to evaluate different dual-Doppler analysis (DDA) methods for retrieving vertical velocity $w$ at grid spacings on the order of $100 \mathrm{~m}$ within a simulated tornadic supercell. Variational approaches with and without a vertical vorticity equation constraint are tested, along with a typical (traditional) method involving vertical integration of the mass conservation equation. The analyses employ emulated radar data from dual-Doppler placements 15,30 , and $45 \mathrm{~km}$ east of the mesocyclone, with volume scan intervals ranging from 10 to $150 \mathrm{~s}$. The effect of near-surface data loss is examined by denying observations below $1 \mathrm{~km}$ in some of the analyses. At the longer radar ranges and when no data denial is imposed, the "traditional" method produces results similar to those of the variational method and is much less expensive to implement. However, at close range and/or with data denial, the variational method is much more accurate, confirming results from previous studies. The vorticity constraint shows the potential to improve the variational analysis substantially, reducing errors in the $w$ retrieval by up to $30 \%$ for rapid-scan observations ( $\leq 30 \mathrm{~s}$ ) at close range when the local vorticity tendency is estimated using spatially variable advection correction. However, the vorticity constraint also degrades the analysis for longer scan intervals, and the impact diminishes with increased range. Furthermore, analyses using 30-s data also frequently outperform analyses using 10-s data, suggesting a limit to the benefit of increasing the radar scan rate for variational DDA employing the vorticity constraint.
\end{abstract}

\section{Introduction}

The ability to retrieve three-dimensional wind fields from velocity observations by two Doppler radars (Armijo 1969) has a wide range of applications. It can be used to study the structure, kinematic properties, and microphysical processes (e.g., hail growth in Ziegler et al. 1983) of convective phenomena, including squall lines (Carbone 1982; Roux et al. 1984; Kessinger et al. 1987), microbursts (Parsons and Kropfli 1990; Lee et al. 1992), heavy rain events (Shao et al. 2004), tropical cyclones (Kosiba and Wurman 2014; Wingo and Knupp 2016), supercells (Brandes 1977; Ray et al. 1975; Frame et al. 2009; Markowski et al. 2012; Calhoun et al. 2013;

Corresponding author: Nathan A. Dahl, dahl_nathan@ou.edu
Atkins et al. 2014), and tornadoes (Marquis et al. 2008, 2012; Brandes 1984a,b; Dowell and Bluestein 1997, 2002a,b; Beck et al. 2006; Wurman et al. 2007; Kosiba et al. 2013; Wienhoff et al. 2018; Markowski et al. 2018). It can also be used to study the planetary boundary layer (Gal-Chen and Kropfli 1984) and inform climate model parameterizations (Ferrell et al. 2014).

The vertical velocity $w$ is of prime interest for such applications. In typical scanning geometries with shallow elevation angles, $w$ is poorly observed and the most difficult wind component to retrieve accurately. A common method of dual-Doppler wind analysis [first described in Brandes (1977)] is to map the radial wind observations to a Cartesian grid, estimate horizontal wind components $u$ and $v$ from the mapped data and an initial guess for $w$, vertically integrate the anelastic mass 
conservation equation to update the $w$ field, and iterate until the solution converges. However, substantial gaps between the lowest level of radar coverage and the surface (e.g., due to beam elevation, Earth curvature, and blockage by surface obstructions) can complicate the use of an impermeable lower boundary condition when integrating the mass conservation equation. Some investigators extrapolate data down to the surface, for example, by setting the divergence within the gap equal to a fraction of the divergence at the lowest radar level (Brandes 1977) or through downward propagation of directional cosine terms (Kosiba et al. 2013). However, explicit vertical integration of the mass conservation equation is prone to error accumulation (e.g., see Ray et al. 1980; Gao et al. 2004), and Ray et al. (1985) and Dowell and Shapiro (2003) showed that the iterative approach may diverge if stability conditions based on the observation geometry are not met.

More recently, variational methods have been explored as a way of avoiding these problems. Gao et al. (1999) and Potvin et al. (2012a) obtained good results in observation system simulation experiments (OSSEs) through minimization of a cost function with weak observation, mass conservation, and smoothness constraints, although the gap between the surface and the lowest level of radar data in those cases was small relative to the depth of the analysis domain. Subsequent OSSEs (Mewes and Shapiro 2002; Shapiro et al. 2009; Potvin et al. 2012b, hereafter PSX12) explored the issue of data scarcity near the ground and found that including a vertical vorticity equation as an additional weak constraint substantially improved the $w$ analysis, particularly for rapid radar scan rates.

As an extension of prior studies, the present study refines the approach detailed in PSX12 and explores the use of higher-resolution grids over a variety of radar ranges and scan intervals. The paper is organized as follows: section 2 gives an overview of data acquisition and analysis methods. Section 3 reports and analyzes the experimental results. Section 4 summarizes the findings and provides recommendations for further inquiry.

\section{Methods and data}

a. "True" wind fields and radar pseudo-observations

Efforts to refine dual-Doppler analysis (DDA) techniques are hindered by a lack of $w$ observations for verification. To circumvent that limitation, this study employs the commonly used OSSE approach (e.g., Clark et al. 1980; Gao et al. 1999; Mewes and Shapiro 2002; Shapiro et al. 2009; Potvin et al. 2012a; PSX12). Output wind and reflectivity fields from a high-resolution Advanced Regional Prediction System (ARPS; Xue et al. 2000, 2001) simulation of the 8 May 2003 Oklahoma City tornadic supercell, described in Xue et al. (2014) and Schenkman et al. (2014) serve as the "true" state. The storm and its environment are modeled on a terrain-following grid with $50-\mathrm{m}$ horizontal spacing and vertical spacing stretched from a minimum of $20 \mathrm{~m}$ at the surface. Initial and boundary conditions are obtained from coarser-grid forecasts employing cycling assimilation of surface, rawinsonde, and radar observations, with progressive downscaling accomplished through oneway grid nesting. The lower boundary is semislip with stability-dependent surface drag (Byun 1990) and local roughness lengths estimated from land-use tables. The simulation includes a period of cyclical tornadogenesis (Fig. 1; see also Fig. 9d of Schenkman et al. 2014), which is selected for analysis here.

The analyses are performed on a $20 \mathrm{~km} \times 20 \mathrm{~km} \times$ $5 \mathrm{~km}$ grid encompassing the intense part of the storm, surrounded by a $26 \mathrm{~km} \times 26 \mathrm{~km} \times 5 \mathrm{~km}$ grid centered at the same location (Fig. 2). The outer grid is used to run provisional retrievals and apply an advection correction procedure (see next section). The $3-\mathrm{km}$ buffer between the boundaries of the analysis grid and the outer grid ensures that all forward and backward trajectories originating within the analysis grid (used in the advection correction procedure) are included in the final analysis, as discussed in PSX12. A pair of emulated radars is placed east of the analysis grid at $2 \mathrm{~m}$ AGL, spaced $30 \mathrm{~km}$ apart (i.e., $15 \mathrm{~km}$ north and south of the grid center) in order to provide a sufficient cross-beam angle at all locations within the grid. To evaluate the effect of range on the results, radar pairs are placed $15 \mathrm{~km}$ (E15), $30 \mathrm{~km}$ (E30), and $45 \mathrm{~km}$ (E45) east of the grid center as shown in Fig. 2. To account for the data becoming more coarse as range increases (i.e., because of beam broadening), the (isotropic) analysis grid spacing $\Delta$ is $125 \mathrm{~m}$ for E15, $250 \mathrm{~m}$ for E30, and $375 \mathrm{~m}$ for E45. The "true" $w$ used for validating each analysis is obtained by smoothing the ARPS output to the corresponding analysis grid using Cressman (1959) interpolation with the cutoff radius set to $\Delta$.

Each volume scan encompasses the outer grid at time intervals $\tau$ ranging from 10 to $150 \mathrm{~s}$. A volume scan consists of a series of conical sweeps at constant elevation angle $\phi$ [i.e., plan position indicators (PPIs)]. A single sweep at $\phi=0^{\circ}$ is performed to ensure data coverage close to ground level [ignoring real-world problems arising from complex beam interactions with the ground, e.g., as described in Snyder and Bluestein (2014)], with subsequent sweeps starting at 




FIG. 1. "True" horizontal winds (vectors) and vertical winds (color fill) at $3 \mathrm{~km}$ above radar level, valid at the analysis time, within the provisional domain for the ARPS simulation. Hatched regions denote areas where the radar reflectivity is less than $5 \mathrm{dBZ}$. Occluded and developing tornado locations are denoted by "T1" and "T2," respectively.

$\phi=0.5^{\circ}$ and increasing in elevation at $1^{\circ}$ increments up to the top of the analysis domain at the edge nearest to the radar. It should be noted that the analysis domain extends only from the surface up to $5 \mathrm{~km}$ AGL to acknowledge realistic limitations on the range of elevation angles that can be sampled using current radar technology when rapid updates are sought at high vertical resolution. For example, observing up to $10 \mathrm{~km}$ AGL (e.g., to include the equilibrium level in a severe storm environment) at $1^{\circ}$ increments from a range of $5 \mathrm{~km}$ (i.e., the approximate distance from the E15 placement to the edge of the analysis grid) would require a volume scan containing more than 60 PPIs, which is well outside the capabilities of current radars for the shorter scan times examined here. (See section 4 for further discussion.)

Radar observations are emulated using the method described in Dahl and Nolan (2018). Each resolution volume is assumed to contain a uniform distribution of ideal scatterers moving precisely with the wind. Thus, the radial wind at each grid point is computed from the air motion as follows:

$$
V_{i}=\left(\mathbf{V}_{i}+w_{t i} \hat{\mathbf{k}}\right) \cdot \mathbf{r}_{i} .
$$

Here, $\mathbf{V}_{i}$ is the true three-dimensional wind at the $i$ th grid point within the resolution volume, $\mathbf{r}_{i}$ is the range vector between the grid point and the radar, and $w_{t i}$ is the hydrometeor terminal velocity calculated from the reflectivity at the grid point using the empirical formula described in Atlas et al. (1973). A simple weighting method [similar to the one described in

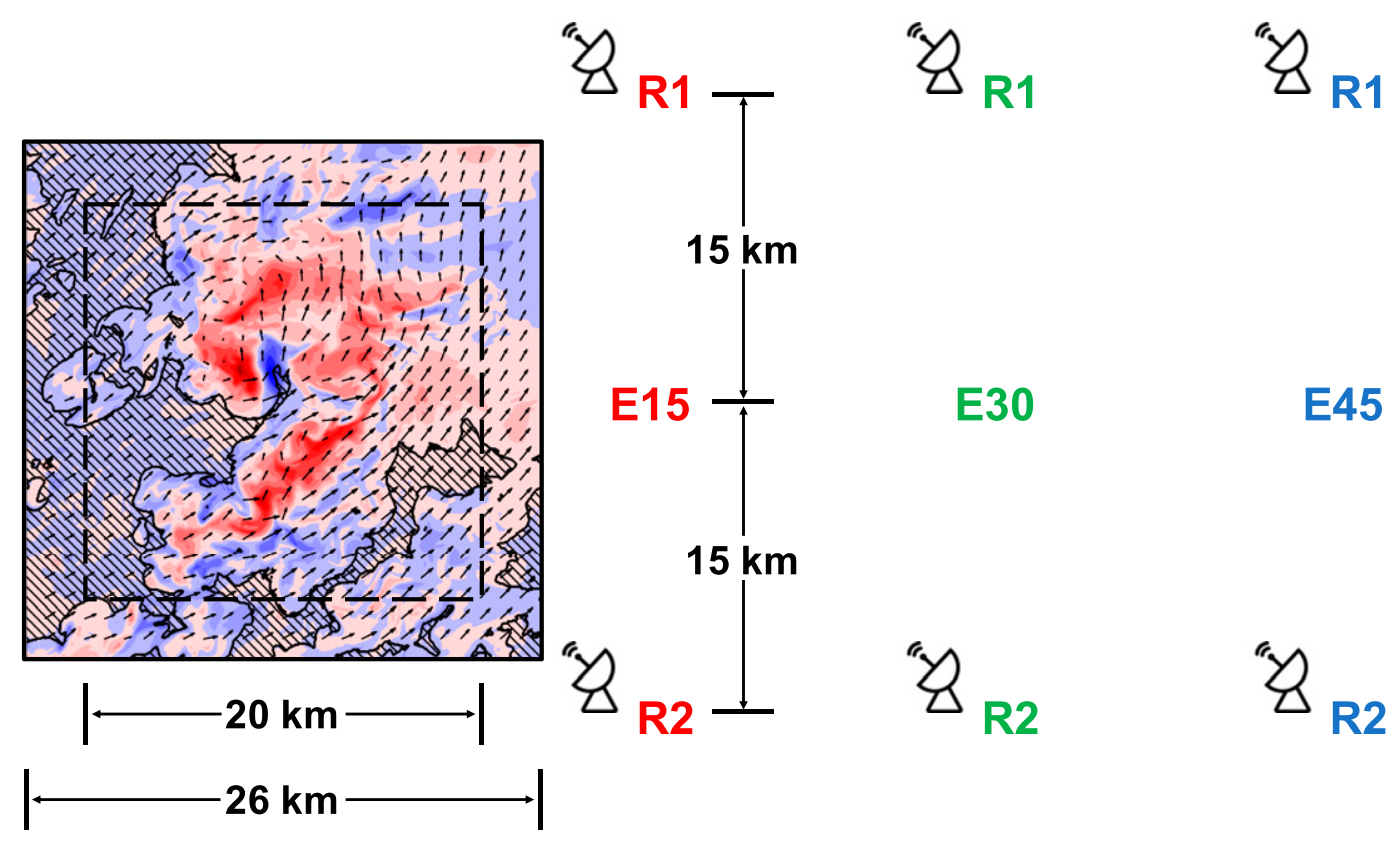

FIG. 2. Summary of virtual radar locations used in this study. North (R1) and south (R2) radars are placed $15 \mathrm{~km}$ (red, E15), $30 \mathrm{~km}$ (green, E30), and $45 \mathrm{~km}$ (blue, E45) east of the center of the provisional domain (solid box) and the analysis domain (dashed box) superimposed on the ARPS simulated vertical velocity field shown in Fig. 1. 
Potvin et al. (2009)] is used to calculate the radial wind observation for each resolution volume:

$$
V_{r}(r, \theta, \phi)=\frac{\sum_{i=1}^{n} B_{i} R_{i} V_{i}}{\sum_{i=1}^{n} B_{i} R_{i}} .
$$

Here, $n$ is the number of grid points in a resolution volume centered at radius $r$, azimuth angle $\theta$, and elevation angle $\phi$. The $R_{i}$ is the range weight, given by

$$
R_{i}=\left\{\begin{array}{ll}
1, & \left|\Delta r_{i}\right|<0.3 d \\
\min \left(\frac{0.5 d-\left|\Delta r_{i}\right|}{0.2 d}, 0\right), & \left|\Delta r_{i}\right| \geq 0.3 d
\end{array},\right.
$$

where $d=100 \mathrm{~m}$ is the range resolution of the radar and $\Delta r_{i}$ is the difference between $\left|\mathbf{r}_{i}\right|$ and $r$. (In other words, the range weight is trapezoidal, set to 1 for an observation whose range differs from the range to the center of the resolution volume by less than $0.3 d=30 \mathrm{~m}$ and decreasing linearly to 0 for an observation for which the range difference is $50 \mathrm{~m}$ or more.) The $B_{i}$ is the beam weight for the $i$ th model grid point, given by

$$
B_{i}=\exp \left\{-8 \ln 2\left[\left(\frac{\theta_{i}}{\theta_{B}}\right)^{2}+\left(\frac{\phi_{i}}{\phi_{B}}\right)^{2}\right]\right\},
$$

where $\theta_{i}$ and $\phi_{i}$ are the differences in radar-relative azimuth and elevation angles (respectively) between $\mathbf{r}_{i}$ and the center of the beam and $\theta_{B}$ and $\phi_{B}$ are the horizontal and vertical half-power beamwidths (set to $\left.1.0^{\circ}\right)$. The altitude of the beam center at a given $(x, y)$ location is calculated using the 4/3 Earth radius model (Doviak and Zrnić 1993). Similar to PSX12, $V_{r}$ observations corresponding to reflectivity $<5 \mathrm{dBZ}$ are screened out, leaving data voids as shown in Fig. 1.

To convert the data from PPIs to the Cartesian grid, the observations are first mapped from $(r, \theta)$ locations to gridded $(x, y)$ locations along each PPI using Cressman interpolation. A spatially constant cutoff radius $r_{c}$ is used, as in PSX12. A cutoff radius roughly equal to the maximum data spacing $D_{\max }(698 \mathrm{~m}$ for $\mathrm{E} 15,901 \mathrm{~m}$ for E30, and $1128 \mathrm{~m}$ for E45) is commonly recommended (e.g., Trapp and Doswell 2000); however, better results are obtained for these experiments (see appendix A) by employing a smaller radius $\left(r_{c}=400 \mathrm{~m}\right.$ for $\mathrm{E} 15,500 \mathrm{~m}$ for $\mathrm{E} 30$, and $600 \mathrm{~m}$ for E45, i.e., slightly greater than $0.5 D_{\max }$ ) and subsequently applying a twopass Leise filter (Leise 1982). Linear vertical interpolation is used to transfer the resulting fields from PPI surfaces to the analysis grid.

\section{b. Variational dual-Doppler analysis formulation and algorithm}

Following PSX12, this study's variational DDA obtains wind component estimates $u^{a}, v^{a}$, and $w^{a}$ by using the Polak-Ribiere conjugate gradient method (Press et al. 1992) to minimize a cost function:

$$
J=J_{O}+J_{M}+J_{V}+J_{S},
$$

where $J_{O}, J_{M}, J_{V}$, and $J_{S}$ are penalty terms for observational, mass conservation, vertical vorticity equation, and smoothness constraints, respectively. Each term is assigned a constant weight denoted by a subscripted $\lambda$. Here, the observational term

$$
J_{O} \equiv \lambda_{O}\left[\sum_{\text {Cart1 }}\left(V_{r 1}^{\mathrm{obs}}-V_{r 1}^{a}\right)^{2}+\sum_{\text {Cart2 }}\left(V_{r 2}^{\mathrm{obs}}-V_{r 2}^{a}\right)^{2}\right]
$$

sums the squared difference between the radial wind observations $V_{r}^{\text {obs }}$ (interpolated to the Cartesian grid) for radars R1 and R2 and the corresponding analyzed radial wind $V_{r}^{a}$. It should be noted that PSX12 sought to account for a finite scan rate by adjusting $V_{r}^{a}$ from the analysis location and time to a shifted location corresponding to the time of observation; since one purpose of the current study is to focus specifically on errors associated with the vertical vorticity equation constraint, any mismatch between the local observation time and the analysis time is neglected in these tests. (This could be characterized as assuming that each scan is instantaneous or, equivalently, that the data are shifted perfectly from the local observation time to the analysis time prior to DDA.)

The terms $J_{M}$ and $J_{V}$ sum the squared residuals on the Cartesian grid from the anelastic mass conservation and anelastic vertical vorticity equations, respectively:

$$
J_{M} \equiv \lambda_{M} \sum_{\text {Cart }}\left(\frac{\partial u^{a}}{\partial x}+\frac{\partial v^{a}}{\partial y}+\frac{\partial w^{a}}{\partial z}+\frac{w^{a}}{\rho} \frac{d \rho}{d z}\right)^{2}, \quad \text { and }
$$

$$
J_{V} \equiv \lambda_{V} \sum_{\text {Cart }}\left[\frac{\partial \zeta}{\partial t}+u^{a} \frac{\partial \zeta^{a}}{\partial x}+v^{a} \frac{\partial \zeta^{a}}{\partial y}+w^{a} \frac{\partial \zeta^{a}}{\partial z}+\left(\frac{\partial v^{a}}{\partial z} \frac{\partial w^{a}}{\partial x}-\frac{\partial u^{a}}{\partial z} \frac{\partial w^{a}}{\partial y}\right)+\zeta^{a} \delta^{a}\right]^{2}
$$


where $\rho(z)=\left(1 \mathrm{~kg} \mathrm{~m}^{-3}\right) \exp (-z / 10 \mathrm{~km})$ is the basestate density profile, $\zeta^{a}=\partial v^{a} / \partial x-\partial u^{a} / \partial y$ is the analyzed vertical vorticity, $\delta^{a}=\partial u^{a} / \partial x+\partial v^{a} / \partial y$ is the analyzed horizontal divergence, and the local vertical vorticity tendency $\partial \zeta / \partial t$ is calculated externally and not updated during the analysis (see later discussion).

The term $J_{S}$ is formulated to minimize small-scale noise in the analysis wind fields and spread observational information into data voids:

$$
\begin{aligned}
J_{S} \equiv & \lambda_{S 1} \sum_{\text {Cart }}\left[\left(\frac{\partial^{2} u^{a}}{\partial x^{2}}\right)^{2}+\left(\frac{\partial^{2} u^{a}}{\partial y^{2}}\right)^{2}+\left(\frac{\partial^{2} v^{a}}{\partial x^{2}}\right)^{2}+\left(\frac{\partial^{2} v^{a}}{\partial y^{2}}\right)^{2}\right]+\lambda_{S 2} \sum_{\text {Cart }}\left[\left(\frac{\partial^{2} u^{a}}{\partial z^{2}}\right)^{2}+\left(\frac{\partial^{2} v^{a}}{\partial z^{2}}\right)^{2}\right] \\
& +\lambda_{S 3} \sum_{\text {Cart }}\left[\left(\frac{\partial^{2} w^{a}}{\partial x^{2}}\right)^{2}+\left(\frac{\partial^{2} w^{a}}{\partial y^{2}}\right)^{2}\right]+\lambda_{S 4} \sum_{\text {Cart }}\left(\frac{\partial^{2} w^{a}}{\partial z^{2}}\right)^{2} .
\end{aligned}
$$

Preliminary tests (not shown) indicate that the best results are obtained in these experiments by weighting all smoothness terms equally as was done in PSX12; that is, $\lambda_{S 1}=\lambda_{S 2}=\lambda_{S 3}=\lambda_{S 4} \equiv \lambda_{S}$. All first-order derivatives in (7) and (8) are calculated using two-point centered differences, and all second-order derivatives in (9) are calculated using three-point centered differences valid over $2 \Delta$.

Similar to PSX12, each of the weights is expressed as the product of a nondimensional tuning parameter and a normalization factor in order to streamline the process of determining optimal settings. The observational constraint weight is normalized by the root-mean-square of the observed radial velocity:

$$
\lambda_{O}=C_{O}\left\{\left(\frac{1}{M_{1}+M_{2}}\right)\left[\sum_{\text {Cart1 }}\left(V_{r 1}^{\mathrm{obs}}\right)^{2}+\sum_{\text {Cart2 }}\left(V_{r 2}^{\mathrm{obs}}\right)^{2}\right]\right\}^{-1},
$$

where $M_{1}$ and $M_{2}$ are the total numbers of radial wind observations (mapped to the Cartesian grid) from radars $\mathrm{R} 1$ and R2 (respectively) and $C_{O}$ is set to 1.0 in all experiments. The mass conservation and smoothness weight normalization factors used in the provisional retrievals (which do not employ the vorticity constraint; see later discussion in this section) are estimated from azimuthal changes in observed radial velocity:

$$
\begin{aligned}
\lambda_{M}^{p}= & C_{M}^{p}\left\{( \frac { 1 } { M _ { 1 } + M _ { 2 } } ) \left[\sum_{\text {Cart } 1}\left(\frac{1}{r_{1}} \frac{\partial V_{r 1}^{\mathrm{obs}}}{\partial \theta}\right)^{2}\right.\right. \\
& \left.\left.+\sum_{\text {Cart2 }}\left(\frac{1}{r_{2}} \frac{\partial V_{r 2}^{\mathrm{obs}}}{\partial \theta}\right)^{2}\right]\right\}^{-1}, \text { and } \\
\lambda_{S}^{p}= & C_{S}^{p}\left\{( \frac { 1 } { M _ { 1 } + M _ { 2 } } ) \left[\sum_{\text {Cart } 1}\left(\frac{1}{r_{1}} \frac{\partial^{2} V_{r 1}^{\mathrm{obs}}}{\partial \theta^{2}}\right)^{2}\right.\right. \\
& \left.\left.+\sum_{\text {Cart } 2}\left(\frac{1}{r_{2}} \frac{\partial^{2} V_{r 2}^{\mathrm{obs}}}{\partial \theta^{2}}\right)^{2}\right]\right\}^{-1} .
\end{aligned}
$$

These formulas are similar to those used in PSX12. The current experiments were performed with tuned provisional parameters $C_{M}^{p}=0.001$ and $C_{S}^{p}=0.0001$ to provide the best possible estimates of the provisional $u$ and $v$ fields.

The $u$ and $v$ fields from the provisional retrievals provide a more direct way of normalizing the weights for the final DDA:

$$
\begin{aligned}
& \lambda_{M}=C_{M}^{f}\left[\left(\frac{1}{N}\right)\left(\sum_{\text {Cart }} \delta^{\mathrm{p} 2}\right)\right]^{-1} \\
& \lambda_{V}=C_{V}^{f}\left\{\left(\frac{1}{N}\right)\left[\sum_{\text {Cart }}\left(\frac{\partial \tilde{\zeta}}{\partial t}+u^{p} \frac{\partial \zeta^{p}}{\partial x}+v^{p} \frac{\partial \zeta^{p}}{\partial y}+\zeta^{p} \delta^{p}\right)^{2}\right]\right\}^{-1},
\end{aligned}
$$

$$
\begin{aligned}
\lambda_{S}= & C_{S}^{f}\left\{( \frac { 1 } { N } ) \sum _ { \text { Cart } } \left[\left(\frac{\partial^{2} u^{p}}{\partial x^{2}}\right)^{2}+\left(\frac{\partial^{2} u^{p}}{\partial y^{2}}\right)^{2}+\left(\frac{\partial^{2} u^{p}}{\partial z^{2}}\right)^{2}\right.\right. \\
& \left.\left.+\left(\frac{\partial^{2} v^{p}}{\partial x^{2}}\right)^{2}+\left(\frac{\partial^{2} v^{p}}{\partial y^{2}}\right)^{2}+\left(\frac{\partial^{2} v^{p}}{\partial z^{2}}\right)^{2}\right]\right\}^{-1}
\end{aligned}
$$

where $N$ is the number of grid points in the analysis domain, $\delta^{p}=\partial u^{p} / \partial x+\partial v^{p} / \partial y$ and $\zeta^{p}=\partial v^{p} / \partial x-\partial u^{p} / \partial y$ are the horizontal divergence and vertical vorticity of the provisional wind field (respectively), and $\partial \tilde{\zeta} / \partial t$ is an estimate of local vorticity tendency (to be discussed). Note that, since the provisional retrievals are specifically tuned to obtain good retrievals of $u$ and $v$ but not $w$, the cost function terms involving $w$ are neglected when calculating the normalization factors. By contrast, the final retrievals are tuned to prioritize accuracy in $w$ over accuracy in $u$ and $v$. Based on those tuning experiments (not shown), the nondimensional parameters for the final DDA are set to $C_{M}^{f}=0.5, C_{V}^{f}=0.002$ (when applicable; see the experiment descriptions below), and $C_{S}^{f}=0.0001$.

As in PSX12, a spatially variable advection correction procedure (ADV) is used to evaluate $\partial \tilde{\zeta} / \partial t$ in many of these experiments. As described in Shapiro et al. (2010a,b), 
ADV tracks the motion of patterns in a scalar field $R$ between two observation times $t_{1}$ and $t_{2}$ by minimizing a cost function with weak constraints based on the frozen turbulence hypothesis (i.e., treating $R$ as conserved along pattern translation trajectories) and smoothness:

$$
\begin{aligned}
J \equiv & \iiint\left[\alpha\left(\frac{\partial R}{\partial t}+U \frac{\partial R}{\partial x}+V \frac{\partial R}{\partial y}\right)^{2}\right. \\
& \left.+\beta\left|\nabla_{h} U\right|^{2}+\beta\left|\nabla_{h} V\right|^{2}\right] d x d y d t .
\end{aligned}
$$

Here, $U$ and $V$ are spatially varying (but steady state) pattern translation components. At each time step between $t_{1}$ and $t_{2}$, forward and backward trajectories are launched from each analysis grid point. The $\alpha$ is a data coverage footprint function set to 1 at a given grid point if data are present at the end of the corresponding backward trajectory at $t_{1}$ and at the end of the corresponding forward trajectory at $t_{2}$, as long as neither trajectory leaves the analysis grid; otherwise, $\alpha$ is set to 0 . The $\beta$ is the ADV smoothing parameter.

In PSX12, $\partial \tilde{\zeta} / \partial t$ is calculated from radar data taken at time $t^{a}$ (which is also the analysis time) and the subsequent scan time $t^{a}+\tau$. First, $U$ and $V$ are obtained by applying ADV to the reflectivity field at those times. Provisional DDA is then used to obtain $u^{p}$ and $v^{p}$ (and, hence, $\left.\zeta^{p}\right)$ at those times. The local vorticity tendency at a gridpoint $\mathbf{x}=(x, y, z)$ at time $t^{a}$ is estimated as

$$
\begin{aligned}
\frac{\partial \tilde{\zeta}}{\partial t}\left(\mathbf{x}, t^{a}\right)= & \frac{\zeta^{p}\left(\mathbf{x}^{*}, t^{a}+\tau\right)-\zeta^{p}\left(\mathbf{x}, t^{a}\right)}{\tau} \\
& -\left.U(\mathbf{x}) \frac{\partial \zeta^{p}}{\partial x}\right|_{\mathbf{x}, t^{a}}-\left.V(\mathbf{x}) \frac{\partial \zeta^{p}}{\partial y}\right|_{\mathbf{x}, t^{a}},
\end{aligned}
$$

where $\mathbf{x}^{*}=[x+\tau U(\mathbf{x}), y+\tau V(\mathbf{x}), z]$. The first term on the right-hand side is an estimate of the intrinsic (i.e., pattern following) evolution of $\zeta^{p}$, and first-order derivatives are calculated using centered differences. Since the analysis is performed at the first observation time, this method constitutes a forward difference approximation and the calculated tendencies are valid at $t^{a}+\tau / 2$ rather than at $t^{a}$. To test the impact of this temporal mismatch, the current study includes one set of experiments (refADV_FD) using the PSX12 forward difference method and another set of experiments (refADV_CD) in which a centered difference approximation for $\partial \zeta / \partial t$ valid at $t^{a}$ is obtained instead, as follows: 1 ) apply $\mathrm{ADV}$ to the reflectivity over the interval from $t^{a}-\tau$ to $t^{a}$ to get pattern translation components $U_{1}$ and $V_{1} ; 2$ ) apply $\mathrm{ADV}$ to the reflectivity over the interval from $t^{a}$ to $t^{a}+\tau$ to get pattern translation components $U_{2}$ and $V_{2}$; and 3) estimate the local vorticity tendency using

$$
\begin{aligned}
\frac{\partial \tilde{\zeta}}{\partial t}\left(\mathbf{x}, t^{a}\right)= & \frac{\zeta^{p}\left(\mathbf{x}_{2}^{*}, t^{a}+\tau\right)-\zeta^{p}\left(\mathbf{x}_{1}^{*}, t^{a}-\tau\right)}{2 \tau} \\
& -\left.\bar{U}(\mathbf{x}) \frac{\partial \zeta^{p}}{\partial x}\right|_{\mathbf{x}, t^{a}}-\left.\bar{V}(\mathbf{x}) \frac{\partial \zeta^{p}}{\partial y}\right|_{\mathbf{x}, t^{a}},
\end{aligned}
$$

where $\mathbf{x}_{1}^{*}=\left[x-\tau U_{1}(\mathbf{x}), y-\tau V_{1}(\mathbf{x}), z\right], \mathbf{x}_{2}^{*}=\left[x+\tau U_{2}(\mathbf{x}), y+\right.$ $\left.\tau V_{2}(\mathbf{x}), z\right], \bar{U}(\mathbf{x})=0.5\left(U_{1}+U_{2}\right)$, and $\bar{V}(\mathbf{x})=0.5\left(V_{1}+V_{2}\right)$.

The refADV_FD and refADV_CD methods assume that the translation of the reflectivity field is closely related to the translation of the vertical vorticity field. These methods also treat $U$ and $V$ for a given trajectory as constant, which is likely to produce larger errors when $\tau$ increases. Another option (the "uvADV" method) is to track the provisional wind components $u^{p}$ and $v^{p}$, rather than the observed reflectivity. In this method, $u^{p}$ and $v^{p}$ valid at $t^{a}-\Delta t$ and $t^{a}+\Delta t$ are obtained from $\mathrm{ADV}$, where $\Delta t=5 \mathrm{~s}$ is the ADV computational time step and $\beta=50 \mathrm{~m}^{2} \mathrm{~s}^{-2}$. The (centered difference) local vorticity tendency estimate from this method is given by

$$
\frac{\partial \tilde{\zeta}}{\partial t}\left(\mathbf{x}, t^{a}\right) \approx \frac{\zeta^{p}\left(\mathbf{x}, t^{a}+\Delta t\right)-\zeta^{p}\left(\mathbf{x}, t^{a}-\Delta t\right)}{2 \Delta t} .
$$

Figure 3 summarizes the algorithm for the uvADV method. First, observations from a pair of radars are mapped to the grids at times $t^{a}-\tau, t^{a}$, and $t^{a}+\tau$. Provisional DDA with the vorticity constraint neglected is then performed on the observations valid at each time to get $u^{p}$ and $v^{p}$. The $u^{p}$ and $v^{p}$ are then advection corrected between $t^{a}-\tau$ and $t^{a}$ and between $t^{a}$ and $t^{a}+\tau$; the result provides $u^{p}$ and $v^{p}$ valid at times $t^{a}-\Delta t$ and $t^{a}+\Delta t$, from which $\zeta^{p}\left(\mathbf{x}, t^{a}-\Delta t\right)$ and $\zeta^{p}\left(\mathbf{x}, t^{a}+\Delta t\right)$ are calculated. The $\partial \tilde{\zeta} / \partial t$ is then estimated from (19). Finally, the observations, $\partial \tilde{\zeta} / \partial t$, and $u^{p}$ and $v^{p}$ valid at the analysis time [used to nondimensionalize the constraint weights; see (13)(15)] are applied in the full DDA to retrieve $u^{a}, v^{a}$, and $w^{a}$.

It should be noted that the cost of ADV is not trivial. Another option for calculating $\partial \tilde{\zeta} / \partial t$ is to simply time difference the vertical vorticity fields obtained from $u^{p}$ and $v^{p}$ at times $t^{a}-\tau$ and $t^{a}+\tau$ :

$$
\frac{\partial \tilde{\zeta}}{\partial t}\left(\mathbf{x}, t^{a}\right) \approx \frac{\zeta^{p}\left(\mathbf{x}, t^{a}+\tau\right)-\zeta^{p}\left(\mathbf{x}, t^{a}-\tau\right)}{2 \tau} .
$$

This "brute force" method (denoted "BF") has the merit of removing any need for ADV. However, the risk of larger errors due to nonlinearity must be considered, particularly for large $\tau$.

\section{c. "Traditional" dual-Doppler analysis algorithm and verification method}

As in Potvin et al. (2012a), analyses employing a retrieval method of the sort described in Brandes [1977; 


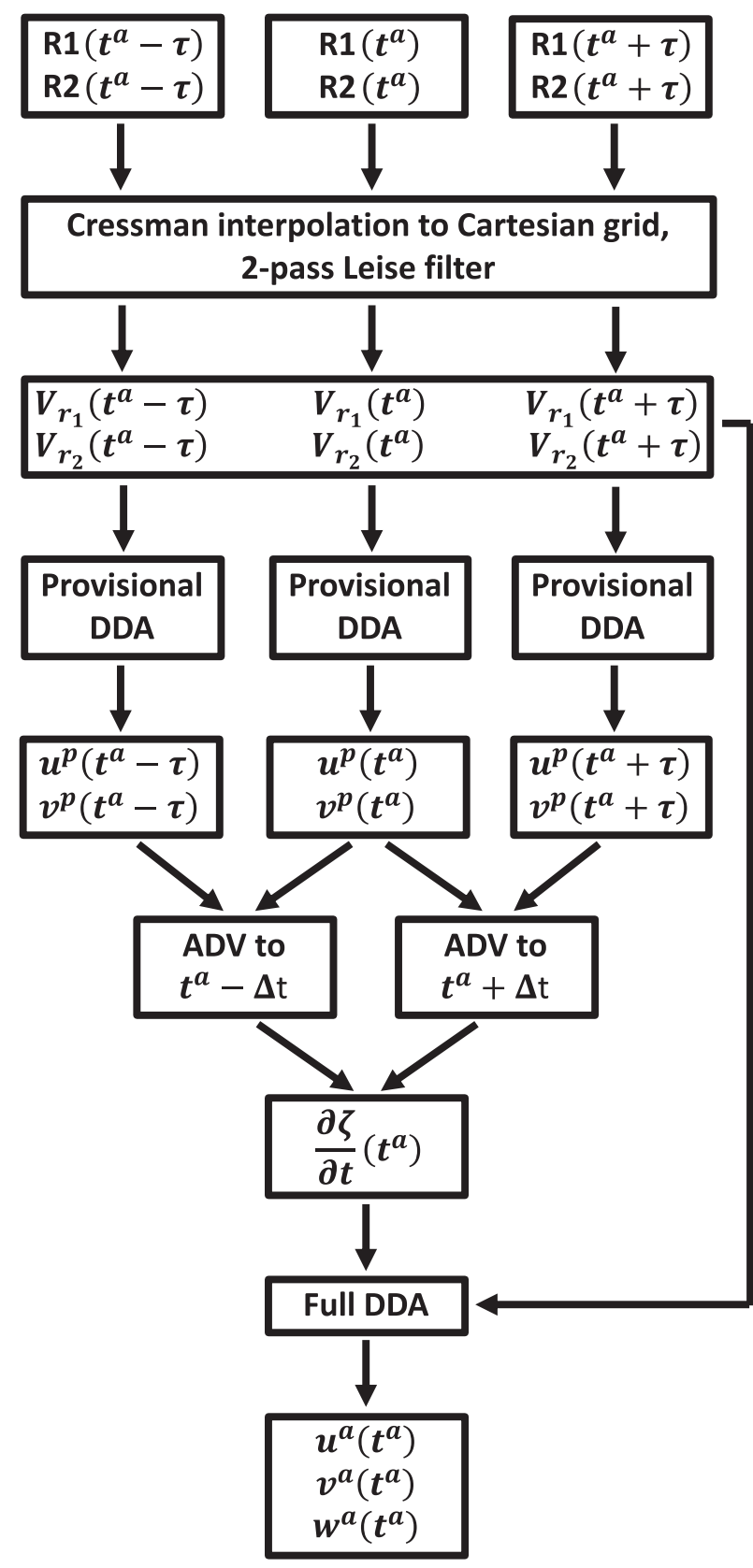

FIG. 3. The uvADV DDA algorithm, with radar input data R1 and R2 and output wind analysis components $u^{a}, v^{a}$, and $w^{a}$. Other terms are defined in the text.

referred to as the "traditional" approach (TRAD)] are used for comparison with the variational method. In the traditional approach, the first guess for $u^{a}$ and $v^{a}$ is obtained from $V_{\mathrm{r} 1}, V_{\mathrm{r} 2}$, and the hydrometeor terminal velocity $w_{t}$ [estimated from reflectivity based on the empirical formula in Atlas et al. (1973)] using (5) in Ray et al. (1980) with $w^{a}$ set to zero. Centered differences are used to estimate the horizontal divergence of this first guess:

$$
\begin{aligned}
\delta(\mathbf{x})= & \frac{u^{a}(x+\Delta x, y, z)-u^{a}(x-\Delta x, y, z)}{2 \Delta x} \\
& +\frac{v^{a}(x, y+\Delta y, z)-v^{a}(x, y-\Delta y, z)}{2 \Delta y} .
\end{aligned}
$$

At the lateral boundaries, where centered differencing is not possible, $\delta$ is set equal to the value at the nearest grid point in the interior of the analysis domain; for example, $\delta(0,0, z)=\delta(\Delta x, \Delta y, z)$. In these experiments, this yields better results than applying zero-gradient lateral boundary conditions for $u^{a}$ and $v^{a}$ or using forward or backward differences (e.g., discretizing over $\Delta x$ instead of $2 \Delta x$ ) to calculate $\delta$ at boundaries. The $\delta$ estimate is then used in a finite difference form of the anelastic mass conservation equation with an impermeable lower boundary to obtain the first guess for $w^{a}$ :

$$
\begin{aligned}
w^{a}(x, y, 0) & =0, \\
w^{a}(\mathbf{x}) & =\frac{\rho(z-\Delta z) w^{a}(x, y, z-\Delta z)-\Delta z \overline{\rho \delta}(x, y, z)}{\rho(z)}, \\
z \geq & \Delta z .
\end{aligned}
$$

Density $\rho$ is calculated from a standard density profile $\rho(z)=\rho_{o} e^{-z / H}$, where $H=10^{4} \mathrm{~m}$. [The specification of $\rho_{o}$ is inconsequential since it cancels out of (22).] The $\overline{\rho \delta}$ is given by

$$
\overline{\rho \delta}(x, y, z)=\frac{\rho(z-\Delta z) \delta(x, y, z-\Delta z)+\rho(z) \delta(x, y, z)}{2} .
$$

The first-guess $w^{a}$ field is combined with radial wind data to update $u^{a}$ and $v^{a}$, which are then used to update $w^{a}$, and the process iterates until the solution converges (here defined as $\left|\Delta\left[w^{a}(\mathbf{x})\right]\right| \leq 0.1 \mathrm{~m} \mathrm{~s}^{-1}$ everywhere, where $\Delta\left[w^{a}(\mathbf{x})\right]$ is the iterative adjustment to $w^{a}$ at location $\left.\mathbf{x}\right)$. A two-pass Leise filter is applied after each iteration to remove noise arising from the finite differencing.

In the common scenario where data are missing near the ground (e.g., because of beam elevation or ground clutter), determining $\delta$ in the layer between the lowest level of data and the surface is problematic. A typical method is to set the mean divergence in this layer equal to a specified fraction $f_{\mathrm{LDL}}$ (e.g., 0.9 in Brandes 1977) of the estimated divergence at the lowest data level. In the present study, the analysis is performed for values of $f_{\mathrm{LDL}}$ ranging from 0 to 4 at intervals of 0.25 ; the analysis with the lowest global root-mean-square error in $w$ ( $w$ RMSE) is selected as the optimal TRAD analysis (see next section) for comparison with the variational DDA results.

It should be noted that this is purely an "upward integration" method, which has been shown elsewhere 
TABLE 1. Attributes of all DDA methods used in this study.

\begin{tabular}{lllccc}
\hline \hline & DDA type & \multicolumn{1}{c}{ Data denial } & Vorticity constraint & ADV & $\begin{array}{c}\text { Time differencing for } \\
\text { vorticity tendency }\end{array}$ \\
\hline D0_TRAD & Traditional & None & No & - & - \\
D1_TRAD & Traditional & Below 1 km AGL & No & - & - \\
D0_VAR_NV & Variational & None & No & - & - \\
D1_VAR_NV & Variational & Below 1 km AGL & No & Reflectivity tracking & Forward \\
D1_VAR_refADV_FD & Variational & Below 1 km AGL & Yes & Reflectivity tracking & Centered \\
D1_VAR_refADV_CD & Variational & Below 1 km AGL & Yes & Wind tracking & Centered \\
D1_VAR_uvADV & Variational & Below 1 km AGL & Yes & None & Centered \\
D1_VAR_BF & Variational & Below 1 km AGL & Yes & & \\
\hline
\end{tabular}

(e.g., Ray et al. 1980; Chong and Testud 1983) to be particularly susceptible to error propagation in the upper levels because of atmospheric density stratification. To reduce this error source, "downward integration" or some combination of the two [e.g., an error-weighted average of upward and downward integration results in Potvin et al. (2012a)] is often employed instead. This requires imposing an upper boundary condition, for example, impermeability if the upper boundary is near the equilibrium level or cloud top where vertical velocities are assumed to be small. [This assumption is often untenable; even when it is valid, Potvin et al. (2012a) report that uncertainties in estimating the horizontal divergence at the upper boundary can still produce substantial errors in the downward integration.] In the current study, such methods are not possible since the upper boundary is in the midlevels where intense vertical motion is present (for the reasons discussed in section 2a); thus, the implications of the upward integration method must be borne in mind when interpreting the results.

To provide a baseline for studying the relative impact of low-level data scarcity, control analyses including all available radar data (denoted D0, indicating "no denial") are first performed for each radar placement using the traditional method (D0_TRAD) or variational DDA with $C_{O}=1.0, C_{M}^{f}=0.5, C_{V}^{f}=0$, and $C_{S}^{f}=0.0001$ (D0_VAR_ $\mathrm{NV}$, where VAR denotes variational DDA and NV indicates that the vorticity constraint is not used). Analyses are then performed with observations below $1 \mathrm{~km}$ AGL excluded (denoted D1 to indicate "data denial below $1 \mathrm{~km}$," roughly equivalent to denying data below $1.5^{\circ}$ elevation at $30-\mathrm{km}$ range). Variational DDA employing the vorticity constraint $\left(C_{V}^{f}=0.002\right)$ with scan interval $\tau=10$, 30,60 , or $150 \mathrm{~s}$ are then performed with $1-\mathrm{km}$ data denial using the reflectivity-tracking ADV methods with forward time differencing (D1_VAR_refADV_FD) and centered time differencing (D1_VAR_refADV_CD), the windtracking ADV method (D1_VAR_uvADV), and the brute force method (D1_VAR_BF). These methods are summarized in Table 1.
Each analysis is evaluated using global and level-bylevel $w$ RMSE based on the truth interpolated to the analysis grid. It is important to note from (7) and (8) that the variational $w$ retrievals (which are based on centered differences) are not valid along the upper boundary. Therefore, the traditional analyses (which evaluate the vertical gradient of $w$ using forward differences) are only performed up to $z=4.5 \mathrm{~km}$, and the error statistics for all analyses are only calculated over the region of dualDoppler coverage from $z=0$ to $z=4.5 \mathrm{~km}$.

\section{Experimental results}

\section{a. Error statistics}

Table 2 summarizes the global $w$ RMSE for the various analyses. There are several items of interest. For the TRAD, the E30 placement produces the best result,

TABLE 2. Global $w$ RMSE $\left(\mathrm{m} \mathrm{s}^{-1}\right)$ for all DDA methods, ranges, and radar scan intervals. The best results for each method employing the vorticity constraint are bolded.

\begin{tabular}{lllll}
\hline \hline & & E15 & E30 & E45 \\
\hline D0_TRAD (optimal) & & 3.65 & 3.14 & 3.75 \\
D1_TRAD (optimal) & & 6.20 & 5.37 & 5.38 \\
D0_VAR_NV & & 2.27 & 3.01 & 3.62 \\
D1_VAR_NV & & 4.16 & 4.68 & 4.74 \\
D1_VAR_refADV_FD & $10 \mathrm{~s}$ & $\mathbf{3 . 4 3}$ & $\mathbf{4 . 3 8}$ & 5.17 \\
& $30 \mathrm{~s}$ & 4.36 & 4.54 & $\mathbf{4 . 7 7}$ \\
& $60 \mathrm{~s}$ & 5.30 & 5.42 & 5.21 \\
& $150 \mathrm{~s}$ & 9.41 & 7.22 & 6.92 \\
D1_VAR_refADV_CD & $10 \mathrm{~s}$ & $\mathbf{3 . 1 1}$ & 3.77 & 4.50 \\
& $30 \mathrm{~s}$ & 3.70 & $\mathbf{3 . 7 6}$ & $\mathbf{4 . 2 7}$ \\
& $60 \mathrm{~s}$ & 4.80 & 4.51 & 4.49 \\
& $150 \mathrm{~s}$ & 8.88 & 7.37 & 6.32 \\
D1_VAR_uvADV & $10 \mathrm{~s}$ & $\mathbf{3 . 2 0}$ & 3.81 & 4.63 \\
& $30 \mathrm{~s}$ & 3.20 & $\mathbf{3 . 7 3}$ & $\mathbf{4 . 3 6}$ \\
& $60 \mathrm{~s}$ & 4.41 & 4.12 & 4.36 \\
& $150 \mathrm{~s}$ & 7.89 & 6.09 & 5.71 \\
D1_VAR_BF & $10 \mathrm{~s}$ & $\mathbf{3 . 5 5}$ & $\mathbf{3 . 8 4}$ & 4.74 \\
& $30 \mathrm{~s}$ & 7.18 & 4.52 & $\mathbf{4 . 4 8}$ \\
& $60 \mathrm{~s}$ & 9.31 & 6.35 & 5.73 \\
& $150 \mathrm{~s}$ & 9.67 & 7.01 & 6.89 \\
\hline
\end{tabular}


E15 D0_TRAD $w(m / s), \max =39.7, \min =-44.2$
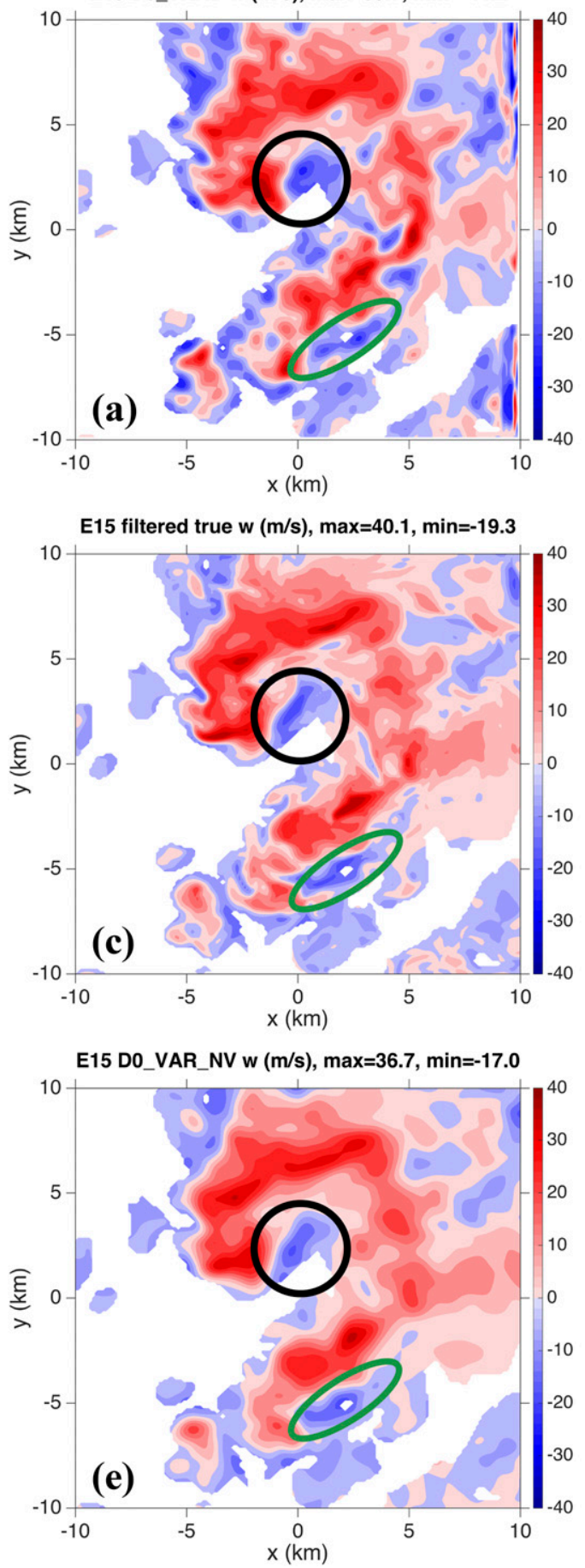

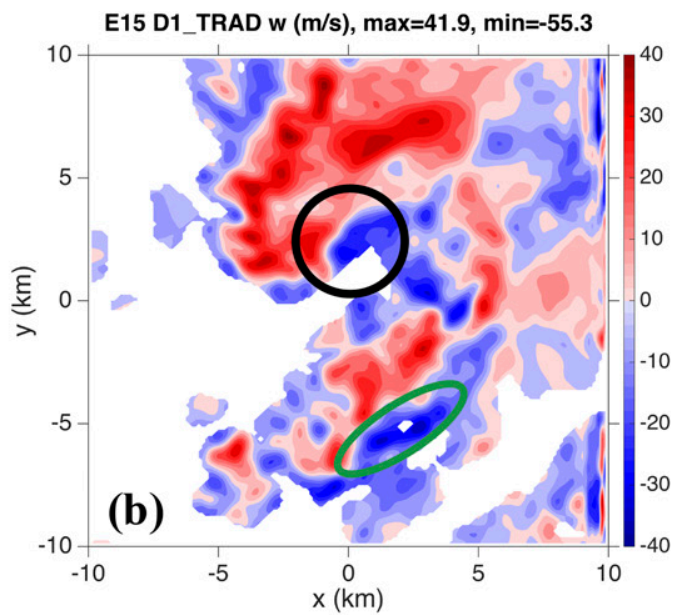

E15 D1_VAR_uvADV $w(\mathrm{~m} / \mathrm{s}), \max =37.9, \min =-16.7$



E15 D1_VAR_NV w $(\mathrm{m} / \mathrm{s}), \max =36.4, \min =-15.4$



FIG. 4. Vertical velocity $w$ fields at $z=4.5 \mathrm{~km}$ AGL valid for the E15 radar placement: (a) traditional retrieval with no data denial; (b) traditional retrieval with 1-km data denial; (c) "true" $w$ filtered to the E15 analysis grid; (d) variational retrieval with $1-\mathrm{km}$ data denial and vorticity constraint employing the uvADV method; (e) variational retrieval with no data denial and no vorticity constraint; and (f) variational retrieval with 1-km data denial and no vorticity constraint. Downdraft regions associated with the occluded tornado and trailing gust front are marked by the black circles and green ovals, respectively. 

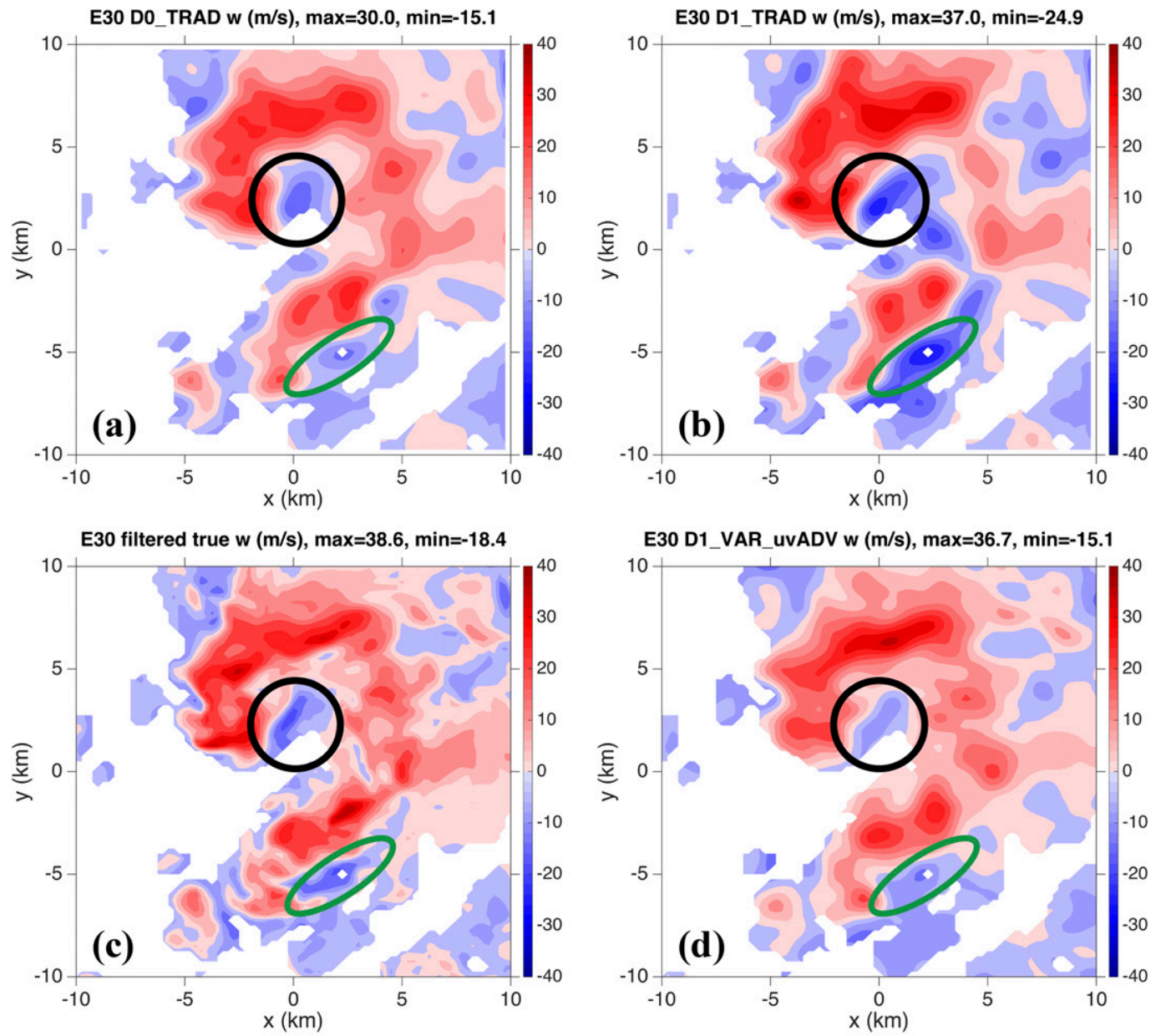

E30 DO_VAR_NV w $(\mathrm{m} / \mathrm{s}), \max =31.6, \min =-18.5$
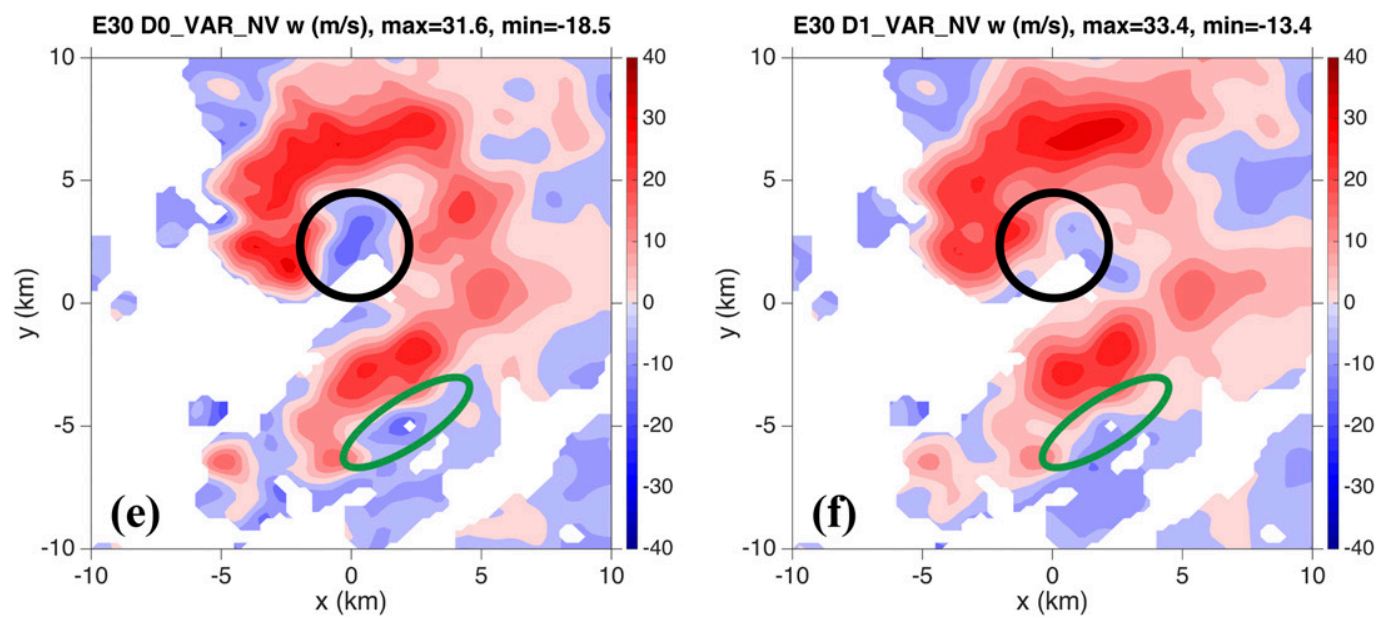

FIG. 5. As in Fig. 4, but for the E30 radar placement.

particularly when there is no denial of low-level data (D0); the $w$ RMSE for the corresponding E15 and E45 placements is $\sim 15 \%-20 \%$ greater. Comparing Figs. $4 \mathrm{a}$ and $4 \mathrm{c}$ and Figs. 5a and 5c, the E15 retrieval has spurious peaks and troughs throughout the domain that are not present in the E30 retrieval, particularly near the eastern boundary where the beam elevation angle becomes large. [In separate experiments applying the traditional method up to $z=5 \mathrm{~km}$, the E15 analyses fail to converge because of violation of a stability 

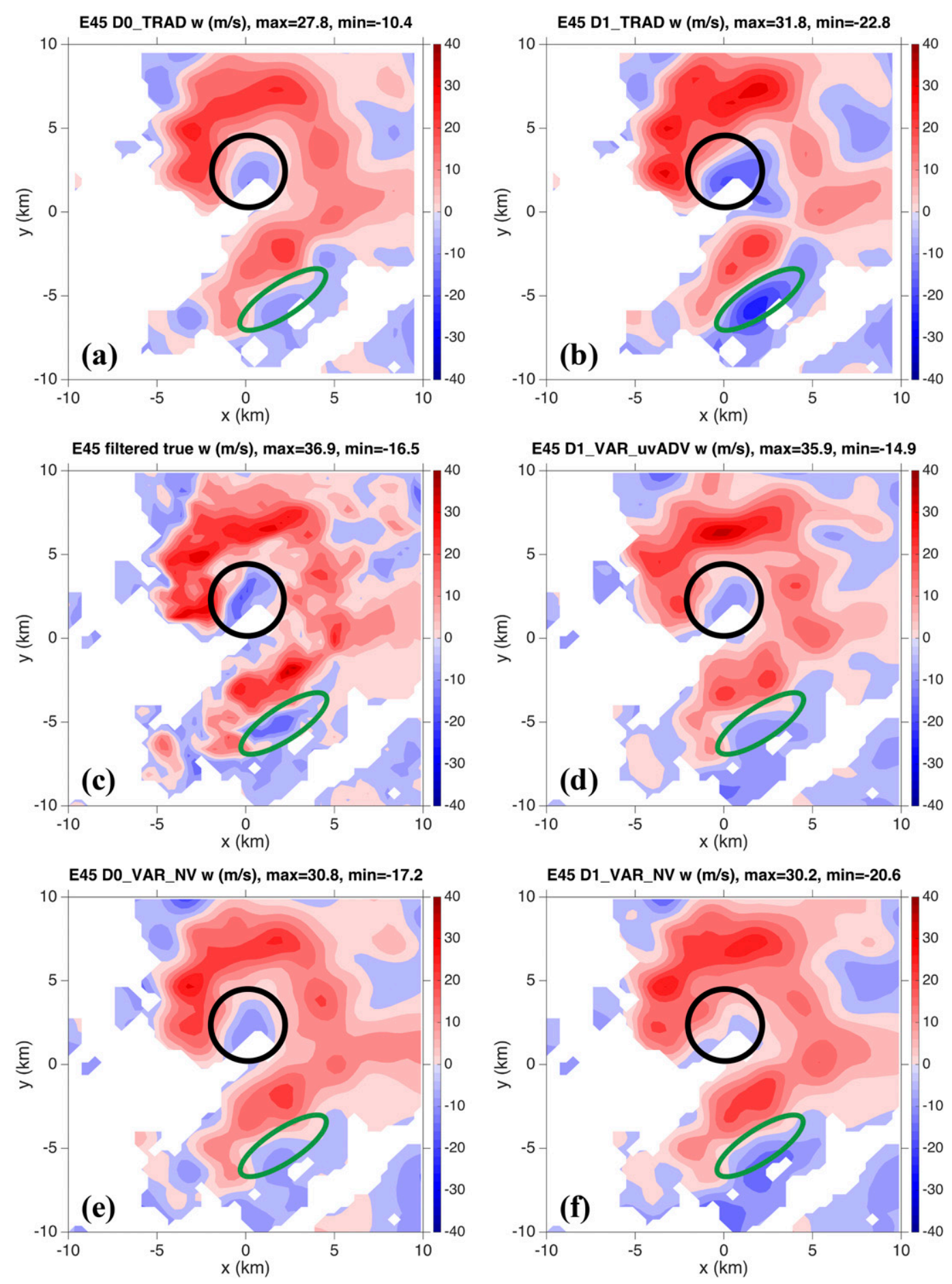

FIG. 6. As in Fig. 4, but for the E45 radar placement.

condition of the kind derived in Dowell and Shapiro (2003).] The error becomes quite large in some of these areas (likely exacerbated by the upward integration method employed, as discussed in section 2c), with the peak downdraft speed in the D1_TRAD analysis exceeding the true peak downdraft speed by more than $35 \mathrm{~m} \mathrm{~s}^{-1}$. As a result, the E15 TRAD $w$ RMSE exceeds the E15 VAR_NV $w$ RMSE by $\sim 60 \%$ for D0 and $\sim 50 \%$ for D1. For the E45 analyses, Fig. 6 shows that the retrieved $w$ is heavily smoothed, as 

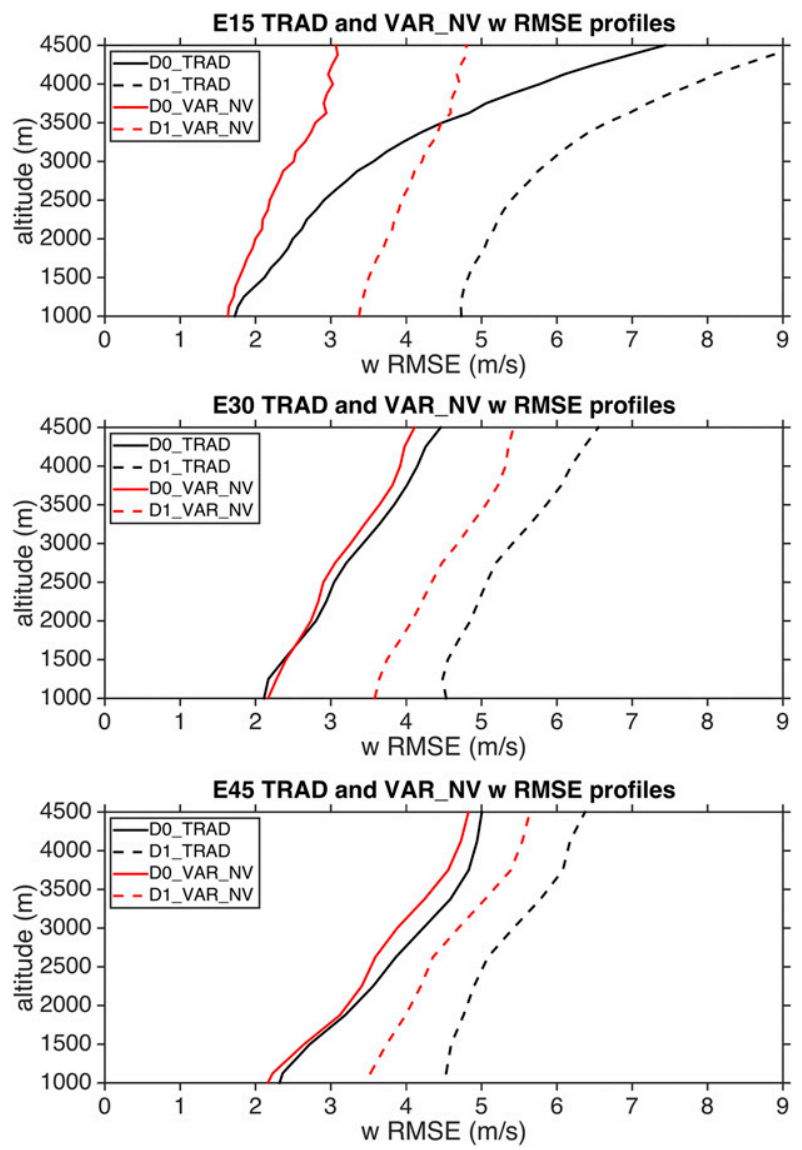

FIG. 7. Vertical profiles of $w$ RMSE for the traditional method (black curves) and the variational method with no vorticity constraint (red curves). Solid curves indicate analyses with no data denial, while dashed curves indicate analyses with 1-km data denial. Results are shown for the (top) E15, (middle) E30, and (bottom) E45 radar placements.

would be expected because of the relative sparseness of the observations.

The $w$ RMSE profiles in Fig. 7 clarify these points further. In all cases, the error for the VAR_NV analysis grows more rapidly with height when the radars are placed farther away, likely because of poorer resolution of the horizontal divergence associated with the more intense vertical motions. TRAD is only slightly less accurate than VAR_NV at all levels for the E30 and E45 placements when no data are missing, similar to the results reported in Potvin et al. (2012a). However, for the E15 placement (a more typical observation range for rapid-scan mobile Doppler radars in severe weather deployments), the error for TRAD becomes very large at upper levels, an indication of the sensitivity of the TRAD method to the coplane elevation angle as described in Dowell and Shapiro (2003). Furthermore, removing the lowest $1 \mathrm{~km}$ of data does substantially more damage to the TRAD analyses than to the VAR_NV analyses. From a comparison of Figs. 4b and 4f, Figs. 5b and 5f, and Figs. $6 \mathrm{~b}$ and $6 \mathrm{f}$, this increase in error appears to stem primarily from spurious amplification of the downdrafts that are present in the true wind field, although there is some overestimation of stronger updrafts at closer ranges as well.

It should be borne in mind that the tabulated TRAD results have the mean divergence optimally specified in the layer between the lowest level of radar data and the surface. Figure 8 shows the fractional increase $\Delta E$ in $w$ RMSE as a function of $f_{\mathrm{LDL}}$, given by

$$
\Delta E\left(f_{\mathrm{LDL}}\right)=\frac{E\left(f_{\mathrm{LDL}}\right)-E_{\min }}{E_{\min }},
$$

where $E$ is the $w$ RMSE for a given specification of $f_{\mathrm{LDL}}$ and $E_{\min }$ is the minimum value of $E$ for a given range and level of data denial. For the D0 retrievals (where the gap between the lowest data level and the surface is $\sim 100 \mathrm{~m}$ ), the optimal $f_{\mathrm{LDL}}$ is $\sim 1$ (similar to Brandes 1977). When the depth of the data void is increased to $1 \mathrm{~km}$ for the D1 retrievals, the optimal $f_{\mathrm{LDL}}$ is $\sim 2.5-3.0$.

However, Fig. 8 generally indicates that the global error is not very sensitive (over a range of "reasonable" values) to the specification of $f_{\mathrm{LDL}}$. A partial explanation for this is that a single value of $f_{\mathrm{LDL}}$ is applied globally in these experiments, whereas the optimal local $f_{\mathrm{LDL}}$ varies widely in space because of the complexity of the wind field; adjusting the global value to reduce errors in some locations increases the errors at other locations, with little net effect on the global statistics. Thus, at least for this set of experiments, there is no global setting that will recover meaningful information when the lowest $1 \mathrm{~km}$ of data are missing. By contrast, the smoothness constraint employed in the data voids for the variational analyses enables a more localized treatment, which provides considerable benefit in this case as long as the constraint is appropriately tuned.

Transitioning to the Table 2 results for retrievals employing the vorticity constraint, obtaining $\partial \tilde{\zeta} / \partial t$ with the brute force method (D1_VAR_BF) applied to 10-s radar data reduces the $w$ RMSE (relative to the error when the vorticity constraint is not used, i.e., D1_VAR_NV) by $15 \%$ for E15 and 18\% for E30. With regard to the increase in $w$ RMSE due to data denial (i.e., relative to the D0_VAR NV results), the increase for D1_VAR_BF is $32 \%$ less than the increase for D1_VAR_NV at the E15 placement and $50 \%$ less at the E30 placement. However, as shown in Fig. 9, the benefit deteriorates rapidly with increased $\tau$ at all levels. Furthermore, there is no benefit from using 10-s data for $\mathrm{E} 45$, whereas the 30-s data provide a small reduction in error (5\% with respect to D1_VAR_NV and $23 \%$ with respect to the increase relative to D0_VAR_NV).

With a few exceptions, Table 2 generally shows that calculating $\partial \tilde{\zeta} / \partial t$ from advection-corrected fields produces 


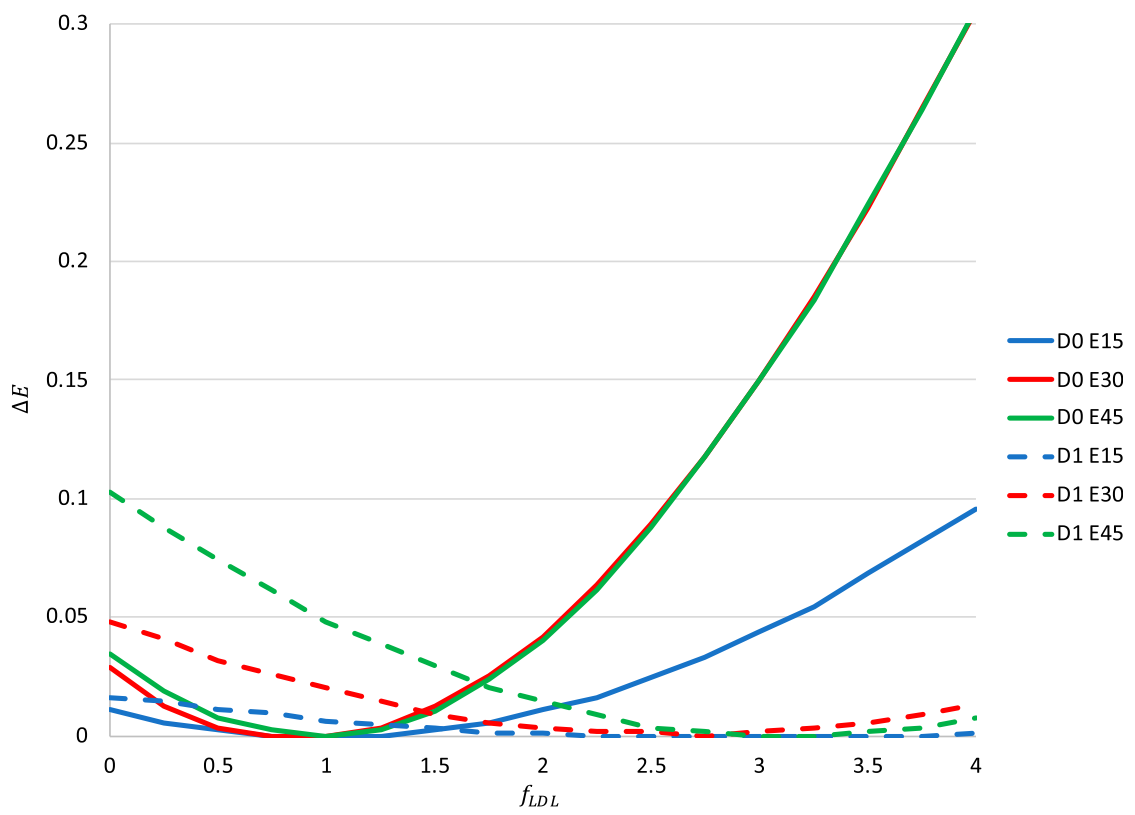

FIG. 8. Fractional departure from optimal $w$ RMSE [ $\Delta E$, defined by (24)] for traditional analyses with no radar data denial (D0) and denial of the lowest $1 \mathrm{~km}$ of data (D1), plotted as a function of the specified divergence in the layer between the lowest level of radar data and the surface. The divergence is specified as a fraction $f_{\mathrm{LDL}}$ of the divergence at the lowest data level.

less error than the brute force method and is more robust to changes in $\tau$. Using forward time differences to obtain $\partial \tilde{\zeta} / \partial t$ (D1_VAR_refADV_FD) shows the smallest improvement; in contrast to the results reported in PSX12, the maximum error reduction for D1_VAR_refADV_FD is small $(<10 \%$ relative to D1_VAR_NV) for the E30 placement and there is no improvement at all for the E45 placement. In fact, for most values of $\tau$, the forward-difference vorticity tendency estimate actually produces a worse analysis than that obtained without the vorticity constraint, similar to what is seen for the D1_VAR_BF results.

It appears that the temporal mismatch $(\tau / 2)$ noted in the previous section is a substantial factor, since the results are almost universally better when a centered time difference is used instead. The $w$ RMSE values for D1_VAR_refADV_CD are substantially less than the values for D1_VAR_refADV_FD for a given placement and $\tau$; they are also less sensitive to changes in $\tau$. The global statistics in Table 2 and the $w$ RMSE profiles in Fig. 10 indicate that the vorticity constraint is beneficial in D1_VAR_refADV_CD up to $\tau=60 \mathrm{~s}$ at longer ranges, although employing the vorticity constraint produces inferior results when $\tau=150 \mathrm{~s}$ regardless of the method used to calculate $\partial \tilde{\zeta} / \partial t$. In contrast, PSX12 reported that the vorticity constraint was beneficial out to $\tau=5 \mathrm{~min}$. This difference is attributed to the higher resolution of the observations and analysis in the present study and the fact that the storm analyzed here is more energetic at small scales (i.e., undergoing cyclical tornadogenesis) than the one in PSX12.

Based on Table 2 and Fig. 11, D1_VAR_uvADV appears to be the most consistent at producing beneficial results; while it is outperformed slightly by D1_VAR refADV_CD for all placements when $\tau=10 \mathrm{~s}$ and for E45 when $\tau=30 \mathrm{~s}$, it is less sensitive to increases in $\tau$. For example, for the E15 placement, D1_VAR_uvADV shows nearly identical benefit for $\tau=10 \mathrm{~s}$ and $\tau=30 \mathrm{~s}$ while the $w$ RMSE in D1_VAR_refADV_CD increases by more than $0.5 \mathrm{~m} \mathrm{~s}^{-1}$ from $\tau=10 \mathrm{~s}$ to $\tau=30 \mathrm{~s}$. This comparative robustness is also seen for the E30 and E45 placements, although it diminishes as the radar range increases. These results suggest that using the reflectivity field to track the evolution of the wind field works well for rapid scans in these experiments, but the mismatch between the reflectivity pattern translation and the wind field pattern translation may become significant beyond $\tau=60 \mathrm{~s}$.

When the vorticity constraint is not used, Figs. 4-6 show examples of additional errors that occur in the variational analysis when the lowest $1 \mathrm{~km}$ of data are missing; for example, compare the downdrafts east of the occluded tornado location (black circles) and south of the trailing gust front (green ovals) in Figs. 4e, 4f, 5e, 5f, 6e, and 6f. Figures $4 d, 5 d$, and $6 \mathrm{~d}$ show that using the vorticity constraint partially restores these details. Comparing Figs. $4 d$ and 4e, Figs. 5d and 5e, and Figs. 6d and 6e shows additional details that are retrieved more accurately with the 

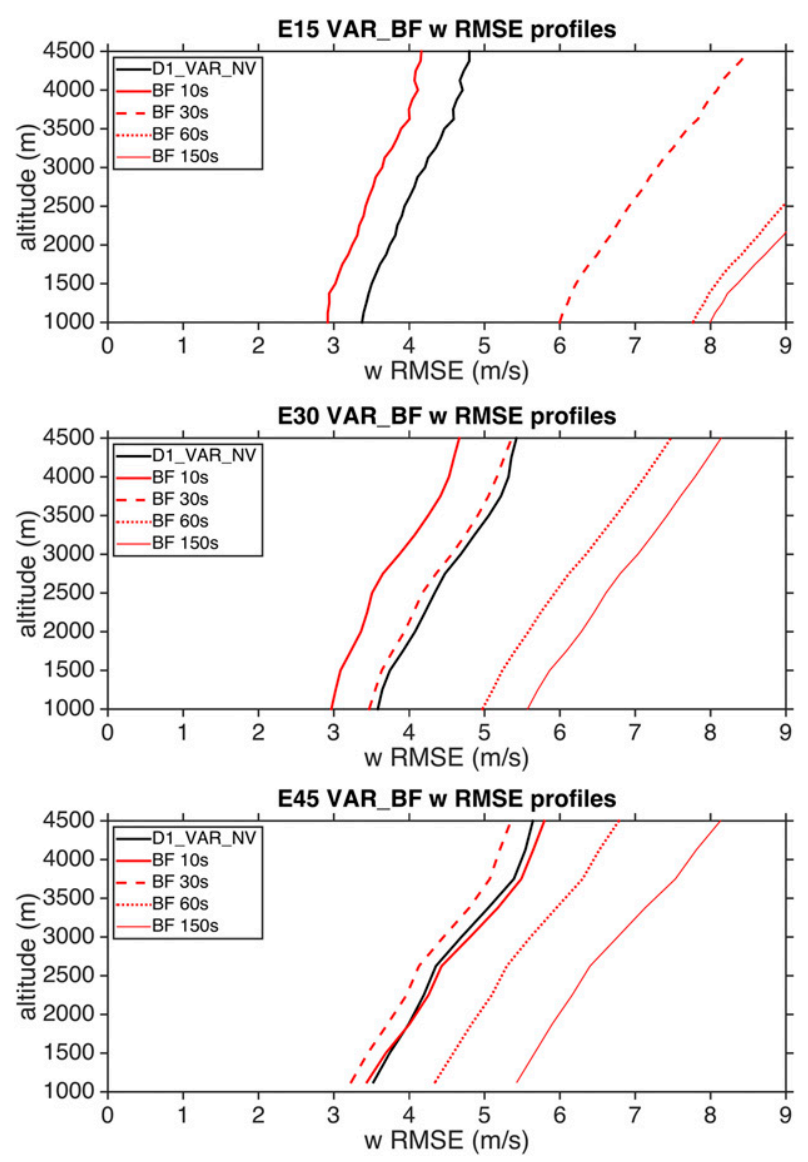

FIG. 9. Vertical profiles of $w$ RMSE for the "brute force" method (red) with 1-km data denial and radar scan intervals of $10 \mathrm{~s}$ (thick solid curve), $30 \mathrm{~s}$ (thick dashed curve), $60 \mathrm{~s}$ (thick dotted curve), and $150 \mathrm{~s}$ (thin solid curve) for the (top) E15, (middle) E30, and (bottom) E45 radar placements. Results obtained without the vorticity constraint are plotted in black.

vorticity constraint even when compared with the variational analysis with no data denial; for example, the maximum retrieved updraft speed is closer to that of the filtered truth for Figs. 4d, 5d, and 6d than for Figs. 4e, 5e, and 6e (particularly in Figs. 5 and 6), and the representation of the smaller updrafts in the northeast corner of the domain is noticeably better in Figs. 4d, 5d, and 6d than in Figs. 4e, 5e, and 6e (particularly in Figs. 4 and 5). However, the utility of the vorticity constraint in reducing general errors decreases in these experiments as the distance from the radar increases and the processes associated with the stronger vertical motions become more poorly resolved.

Surprisingly, when using the vorticity constraint, the best results are frequently not seen where $\tau$ is the smallest. Even for the most simplistic approach with no ADV step (D1_VAR_BF), the relative benefit of small $\tau$ varies with radar range; $\tau=10 \mathrm{~s}$ gives much better results than $\tau=30 \mathrm{~s}$ for the E15 placement, but the

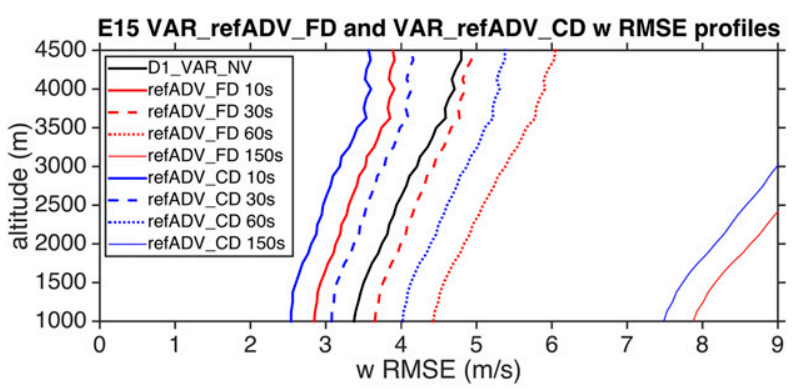

E30 VAR_refADV_FD and VAR_refADV_CD w RMSE profiles

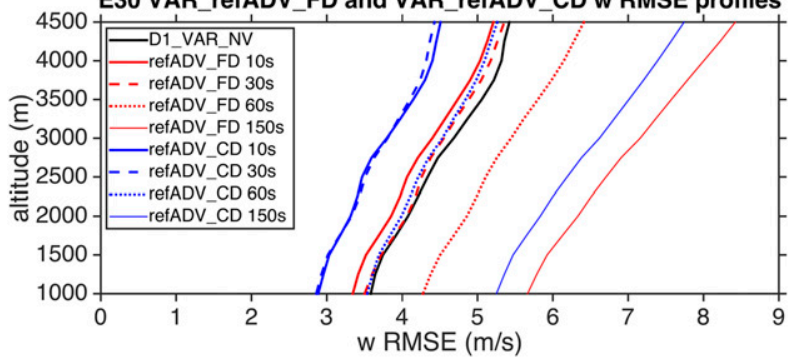

E45 VAR_refADV_FD and VAR_refADV_CD w RMSE profiles

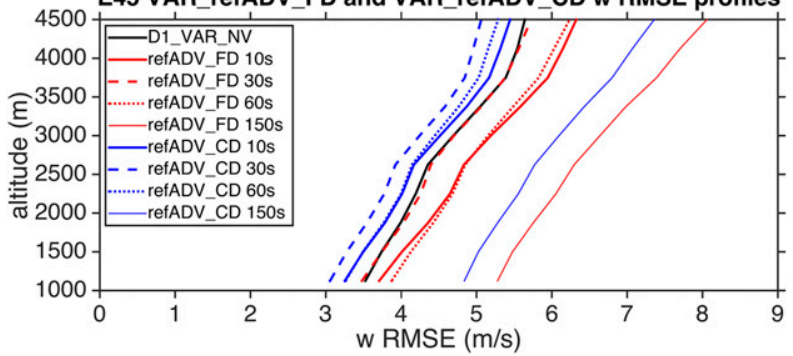

FIG. 10. As in Fig. 9, but for the forward-difference, reflectivity tracking ADV method described in PSX12 (red) and a centereddifference method adapted from it (blue).

difference is greatly reduced for E30 and actually reverses for E45. This issue is examined in further detail in the next section.

\section{b. Impact of scan interval on global error statistics}

When using finite differences to solve time-dependent differential equations, it is commonly expected that a smaller time step $\tau$ will produce a more accurate solution. However, this is not necessarily the case, particularly when both spatial and temporal derivatives are discretized; for example, Falcone and Ferreti (1998) found that the upper bound for the total discretization error for a class of semiLagrangian advection schemes is $C_{1} \tau^{p}+C_{2} \Delta x^{q} / \tau$, where $C_{1}$ and $C_{2}$ are dimensional constants, $\Delta x$ is the space step (e.g., the analysis grid spacing) and $p$ and $q$ are the orders of the space and time discretization schemes respectively. In such cases, for a given $\Delta x$, there is an "optimal" value of $\tau$ below which the total error may actually increase.

Estimating $\partial \zeta / \partial t$ appears to pose a similar problem. For example, consider the brute force estimate given in (20). As noted previously, the $w$ RMSE values in Table 2 
E15 VAR_refADV_CD and VAR_uvADV w RMSE profiles
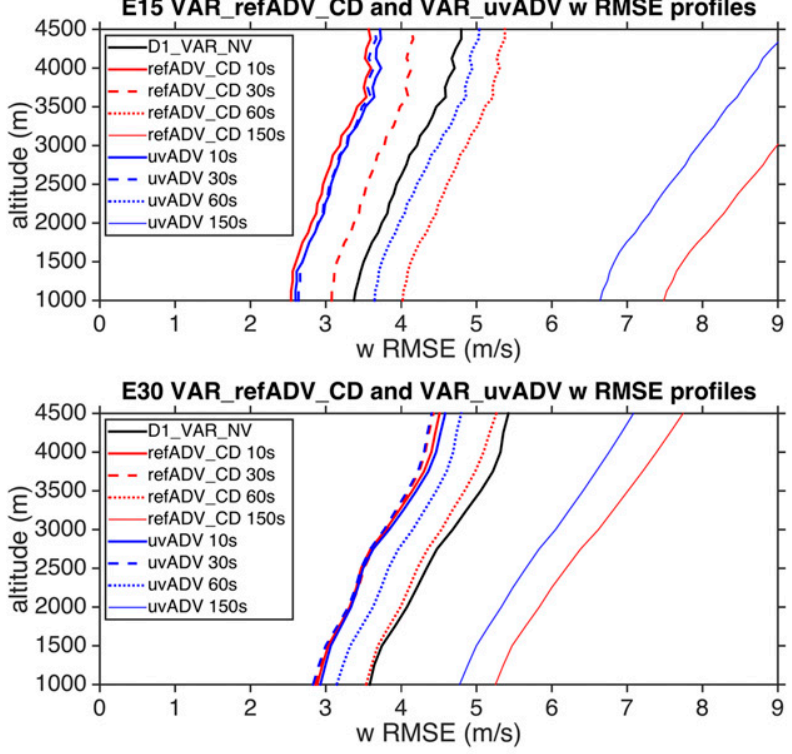

E45 VAR_refADV_CD and VAR_uvADV w RMSE profiles

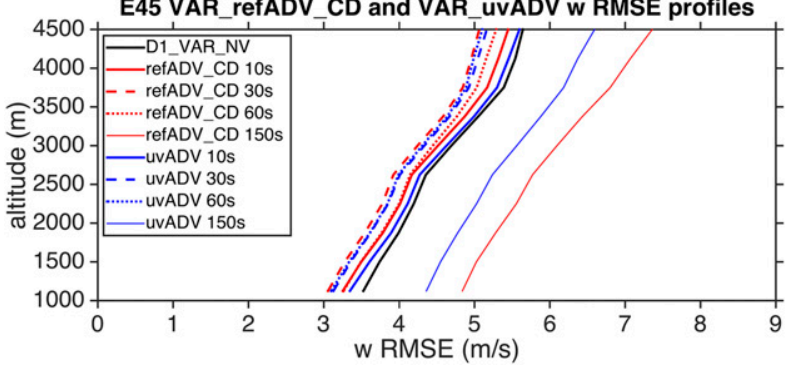

FIG. 11. As in Fig. 9, but for the centered-difference, reflectivity-tracking ADV method (red) and the wind-tracking ADV method (blue).

show that the relative benefit of using 10-s radar data instead of 30-s radar data for the brute force method decreases as the distance from the radar (and hence, in these experiments, the observation spacing $\Delta x_{o}$ and the analysis grid spacing $\Delta x_{a}$ ) increases, to the point that the 10 -s results are worse than the 30 -s results for the E45 placement. This suggests a relationship between $\Delta x_{o}$, $\Delta x_{a}$, and the optimal value of $\tau$.

Rigorous evaluation of this optimal $\tau$ is complicated by the intricacies of the wind field, inaccuracies in the radar observations, and the fact that the vorticity fields at $t^{a}-\tau$ and $t^{a}+\tau$ are calculated from provisional retrievals. These factors constitute separate error sources that are difficult to evaluate analytically. However, to illustrate the basic point, consider a case in which the fluctuation of the $v$ component of the wind is represented by a one-dimensional wave propagating in the $x$ direction at a constant speed, represented as

$$
v(x, t)=V_{o} e^{r t} \cos \left[\frac{2 \pi}{L}(x-U t)\right] .
$$

Here, $V_{o}$ is the initial amplitude of the wave, $r$ is the rate at which the wave amplifies or decays, $L$ is the wavelength, and $U$ is the phase speed. The wave is observed perfectly at a spatial interval $\Delta x_{o}$ and a time interval $\tau$, and the observations are mapped to a grid with spacing $\Delta x_{a}$ using Cressman interpolation with a cutoff radius $r_{c}$ with a two-pass Leise filter subsequently applied, similar to the method described in section 2. Unbiased random noise based on the global RMSE values of the provisional retrievals described in section $2 \mathrm{~b}\left(v^{p}\right.$ RMSE) is then applied to obtain the analyzed field $v^{a}$. Finite differencing of $v^{a}$ is then used to estimate the local tendency of the gradient of the filtered truth $v_{f}$. (The gradient of $v_{f}$ may be thought of as the filtered vertical vorticity component $\partial v_{f} / \partial x$.) The normalized global RMSE of the local gradient tendency estimate is then calculated as a function of $\Delta x_{a}, r_{c}, v_{p}$ RMSE, $\Delta x_{o}, U, r, L$, and $\tau$; a full description of the method for these calculations is given in appendix B.

For comparison with the experimental results, the error for each radar placement is evaluated using the corresponding analysis grid spacing $\Delta x_{a}$ (125 m for E15, $250 \mathrm{~m}$ for E30, and $375 \mathrm{~m}$ for E45), observation Cressman radius $r_{c}(400 \mathrm{~m}$ for $\mathrm{E} 15,500 \mathrm{~m}$ for $\mathrm{E} 30$, and $600 \mathrm{~m}$ for $\mathrm{E} 45)$, and $v_{p}$ RMSE (1.5 m s ${ }^{-1}$ for E15, $2.1 \mathrm{~m} \mathrm{~s}^{-1}$ for E30, and $2.6 \mathrm{~m} \mathrm{~s}^{-1}$ for E45). The $\Delta x_{o}$ is specified as the mean azimuthal spacing between radar observations within the analysis domain ( $370 \mathrm{~m}$ for E15, $585 \mathrm{~m}$ for E30, and $828 \mathrm{~m}$ for E45). The advection correction tests give a typical pattern translation speed $U \sim 20 \mathrm{~m} \mathrm{~s}^{-1}$. Waves with steady $\left(r=0 \mathrm{~s}^{-1}\right)$ and growing (time-scale $1 / r=60 \mathrm{~s}$ ) amplitudes are considered; note that, because a centered difference is used for the time derivative, the RMSE will be the same for a given time scale whether the wave is growing or decaying. With these parameters set, the behavior of the global RMSE for a given radar placement can be examined as a function of $L$ and $\tau$.

For the simulated supercell used here, visual inspection of the true wind fields indicates a typical length scale $\sim 1 \mathrm{~km}$ for fluctuations in $\zeta$; Fig. 12 shows the normalized global RMSE of the estimate of the local gradient tendency [relative to the analytical value given by (B6)], evaluated using either a steady-amplitude or an amplifying wave with a wavelength from 0.5 to $2.5 \mathrm{~km}$. For a given wavelength, the error generally oscillates as a function of $\tau$, with the troughs and peaks (corresponding to the "optimal" and "most suboptimal" values of $\tau$ ) shifting to longer scan intervals as the wavelength increases. Changing the wave amplitude over time increases the oscillations in the RMSE, to the point that the secondary minimum for longer wavelengths at $\tau \sim 150 \mathrm{~s}$ becomes prominent for closer radar placements. On the other hand, the E30 and E45 plots 

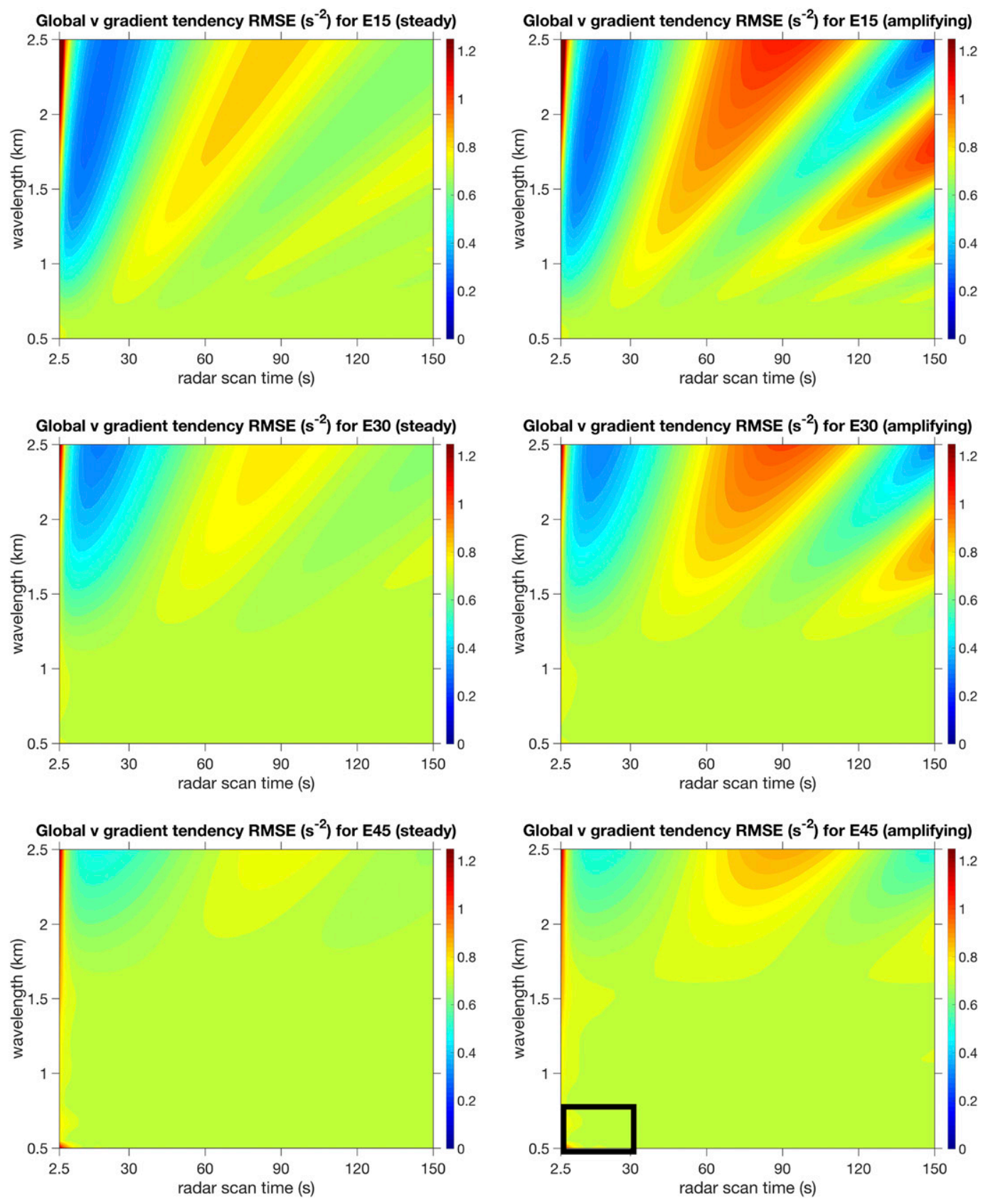

FIG. 12. Normalized global RMSE in estimated gradient tendency of $v$ as defined in (25), as a function of wavelength and scan interval $\tau$, for (left) steady-amplitude waves or (right) waves amplifying with a scale time $1 / r=$ $60 \mathrm{~s}$, where $r$ is the rate of growth for radars placed (top) 15, (middle) 30, and (bottom) $45 \mathrm{~km}$ east of the analysis location. The black box delineates the region plotted in Fig. 13.

suggest that this oscillation is substantially reduced for wavelengths $<4 \Delta x_{a}$. In that range, random errors arising from efforts to analyze poorly resolved features become substantial when $\tau$ becomes small; for example, the $\tau=10 \mathrm{~s}$ error is larger than the $\tau=30 \mathrm{~s}$ error for wavelengths near $0.5 \mathrm{~km}$ observed with the $\mathrm{E} 45$ placement (see Fig. 13).
The secondary minima near $\tau=150 \mathrm{~s}$ in Fig. 12 raise the possibility of good results being obtained with larger $\tau$ even when features are rapidly evolving, as long as those features are large enough to be well resolved by the observations. However, it is necessary to know the characteristics of the wind field a priori to assess whether this possibility exists. For instance, using $\tau=150 \mathrm{~s}$ 


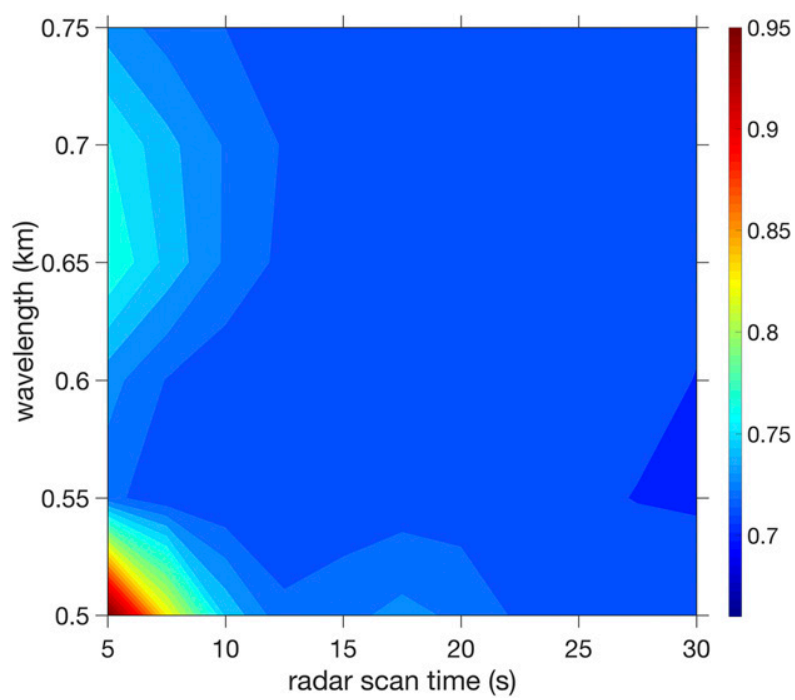

FIG. 13. As in Fig. 12, but for the boxed region. Note that the color scale has been adjusted to focus on values of interest.

produces worse results if the translation speed $U$ changes from 20 to 10 or $30 \mathrm{~m} \mathrm{~s}^{-1}$, as seen by comparing the upperright panel in Fig. 12 with the RMSE plots in Fig. 14. In the absence of prior information regarding $U$, a value of $\tau$ between 10 and 30 s seems to be a comparatively "safe" choice, and the results in Table 2 bear that out. Overall, it is apparent from these tests that the issue of finding an optimal scan interval for employing the vorticity constraint in a given situation is more complicated than one might expect, affected not only by the placement and scanning characteristics (e.g., beamwidth) of the radars but also by the size, translation speed, and intrinsic evolution of the features being observed.

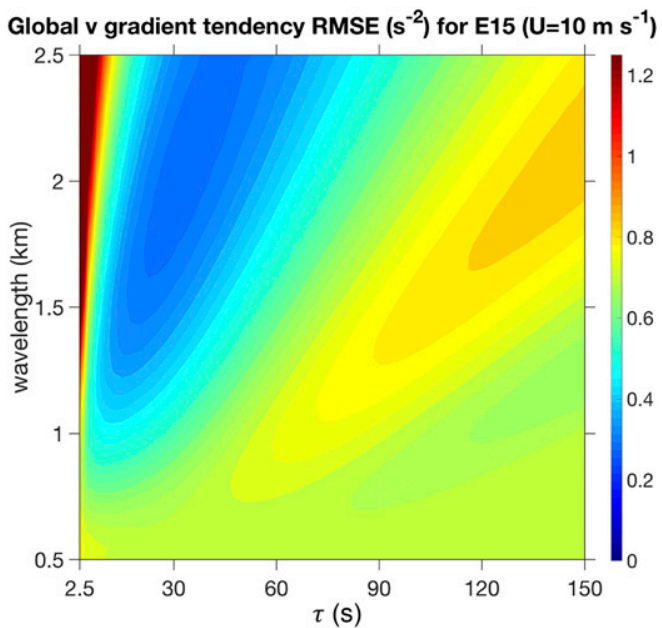

\section{Summary and conclusions}

This study uses an OSSE approach to evaluate different dual-Doppler analysis (DDA) techniques for retrieving vertical velocity from rapid-scan radar observations of a simulated tornadic supercell from a variety of ranges, with radars placed 15,30 , and $45 \mathrm{~km}$ east of the analysis grid center. Emphasis is placed on circumstances where substantial amounts of boundary layer data are missing. These techniques are summarized in Table 1 . Along with the commonly used method of vertically integrating the anelastic mass conservation equation (a TRAD method), variational methods employing weak constraints involving mass conservation, smoothness, and (for some analyses) a vertical vorticity equation are tested. For the variational method employing the vorticity constraint, different methods for estimating the local vertical vorticity tendency are also tested: local centered time differencing of provisional horizontal wind retrievals at times adjacent to the analysis time (VAR_BF), forward time differencing based on trajectories obtained from advection correction of reflectivity fields obtained at the analysis time and the next scan time (described in PSX12 and denoted VAR_ refADV_FD), a centered time difference version of the previous method (VAR_refADV_CD), and centered differencing based on advection correction of provisionally retrieved horizontal wind components (VAR_uvADV).

In this test case, the traditional method performs nearly as well as the variational method with no vorticity constraint (VAR_NV) at longer ranges when data exist within $\sim 100 \mathrm{~m}$ of the ground (i.e., the experiments denoted "D0"). The computational expense of the traditional method is also much less than that of the variational method. However, at a closer range more

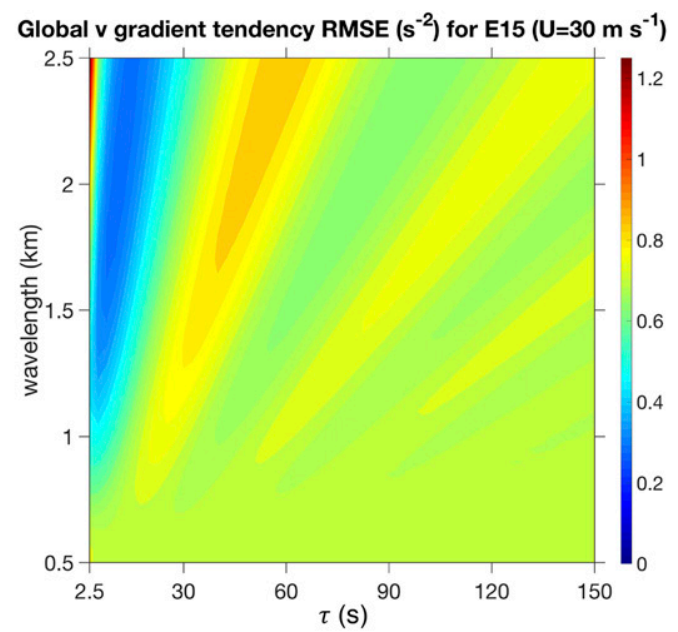

FIG. 14. As in Fig. 12, but for steady-amplitude waves with translation speeds $U=$ (left) 10 and (right) $30 \mathrm{~m} \mathrm{~s}^{-1}$ for radars placed $15 \mathrm{~km}$ east of the analysis location. 
typical of mobile radar observations, the accuracy of the traditional analysis deteriorates rapidly with height (particularly above $\sim 3 \mathrm{~km}$ AGL) and stability problems (see Dowell and Shapiro 2003) are noted if the analysis is attempted up to $5 \mathrm{~km}$ AGL. Furthermore, the variational method is less sensitive to loss of near-surface data, presumably because the smoothness constraint in the variational method provides a more flexible, localized way of approximating conditions in data voids.

The results from variational methods employing the vorticity constraint are mixed. On the one hand, there is evident potential for considerable improvement over the VAR_NV results when substantial amounts of low-level data are missing, with the error due to data denial reduced by $50 \%$ in some instances. However, the benefit is noticeably less at longer ranges, and it is unclear in those instances whether the benefit justifies performing the provisional retrievals and advection correction required to obtain the local vorticity tendency estimate, the computational expense of which can increase the time required to run the full DDA by a factor of 2-3 depending on the grid resolution. It should also be noted that the current vorticity constraint described in (8) does not include any treatment of subgrid mixing, which may be more of a factor at longer ranges as the observations and analysis grid coarsen. Furthermore, it is clear that using an incorrect estimate for the local vorticity tendency may harm the analysis relative to what would be obtained by not using the vorticity constraint at all. For this simulated storm at these resolutions, accurately estimating the local vorticity tendency requires a good method (with the VAR_refADV_CD and VAR_uvADV methods clearly outperforming the VAR_ BF and VAR_refADV_FD methods) and radar scans every $10-30 \mathrm{~s}$; the vorticity constraint is useless for the 150-s scan interval, regardless of method.

It should also be noted that dual-Doppler coverage from the surface to $5 \mathrm{~km}$ AGL requires two radars scanning at elevation angles ranging from near $0^{\circ}$ up to $40^{\circ}$ for the E15 placement. Sampling this span at $\sim 1^{\circ}$ increments (as emulated in this study) within the required time frame would be very difficult using existing radars. Such tasks are becoming more feasible because of advancements in radar technology; for example, the Atmospheric Imaging Radar (AIR; Isom et al. 2013) transmits simultaneously over a $20^{\circ}$ field of view and theoretically could cover the required volume in two sweeps. However, determining an optimal scan time (for the purposes of the DDA method presented here) is also complicated by results demonstrating that more frequent observations may actually harm finite-difference local vorticity tendency estimates in some instances.

In summary, the variational DDA method with the vorticity constraint shows promise for reducing errors in high-resolution vertical velocity retrievals from radar observations of severe convective storms, in agreement with the coarser-resolution results reported in previous studies. Additional cases should be examined to test the generality of these findings. If these results can be generalized, substantial challenges remain for both acquiring the needed data and refining the analysis method (e.g., by finding a way to include subgrid mixing in the vorticity constraint), although it is anticipated that continued advancements will reduce those challenges in the near future.

Acknowledgments. This work was supported by the National Science Foundation through Grant AGS1623626. The authors thank Daniel Betten for assistance with the variational dual-Doppler analysis code, Amy McGovern and Brittany Dahl for providing additional simulation data used to update the code and test the experimental methods, and the anonymous reviewers whose comments helped clarify and refine the presentation of these results.

\section{APPENDIX A}

\section{Radar Data Objective Analysis Using Cressman Interpolation with a Leise Filter}

Objective analysis of radar data is complicated by the spherical geometry of the scans and the consequent spatial variations in observation density. As described by Trapp and Doswell (2000), Cressman (1959) interpolation offers a comparatively sharp response function and is therefore attractive when trying to retain finescale details, but selection of an appropriate Cressman radius is challenging. First, a smaller radius may produce discontinuities in the analyzed fields, whereas a larger radius may smooth them excessively. Second, the response function oscillates when the wavelength of the observed phenomenon is small, producing undesirable sidelobes and consequent phase shifts in the interpolated fields; the wavelength range containing these sidelobes is greater when a larger Cressman radius is used.

The presence of these artifacts at small wavelengths suggests a possible method of mitigation, namely, applying the filter described by Leise (1982) to the results of the Cressman analysis. As noted by Cai (2005), the Leise filter has a cutoff wavelength of $2^{n} \Delta$ and reduces wavelengths of $2^{n+1} \Delta$ by $\sim 24 \%$, where $n$ is the number of filter passes and $\Delta$ is the grid spacing $(125 \mathrm{~m}$ for the E15 placement, $250 \mathrm{~m}$ for E30, or $375 \mathrm{~m}$ for E45). Thus, a twopass Leise filter eliminates wavelengths $\leq 4 \Delta$, which is on the order of the maximum spacing $D_{\max }$ of the observations for the $\mathrm{E} 15\left(4 \Delta=500 \mathrm{~m}, D_{\max }=698 \mathrm{~m}\right)$, 
(a)

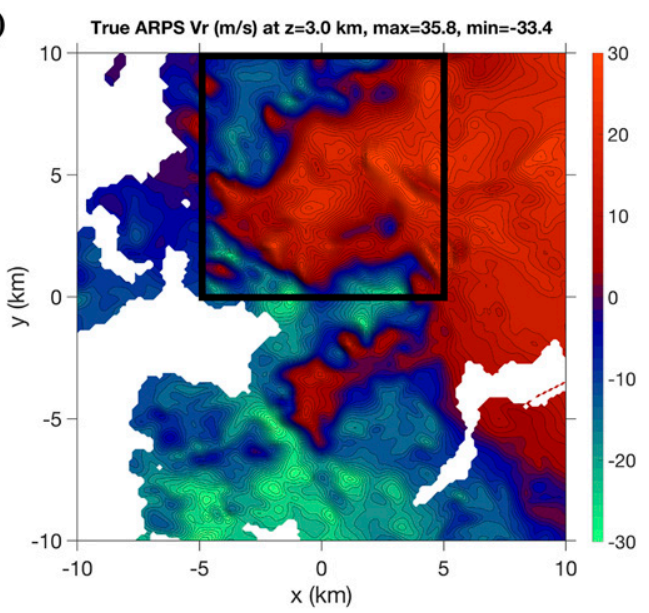

(c) $800 \mathrm{~m}$ Cress, no Leise $\operatorname{Vr}(\mathrm{m} / \mathrm{s})$ at $\mathrm{z}=3.0 \mathrm{~km}, \max =29.7, \mathrm{~min}=-28.4$

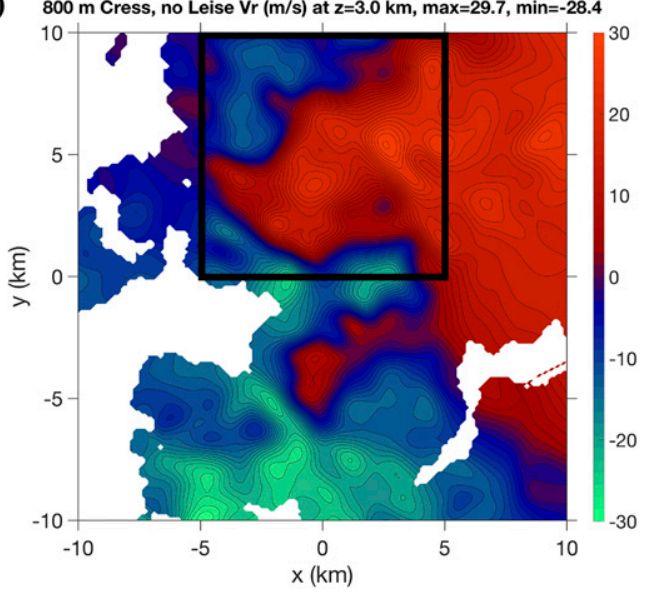

(b)

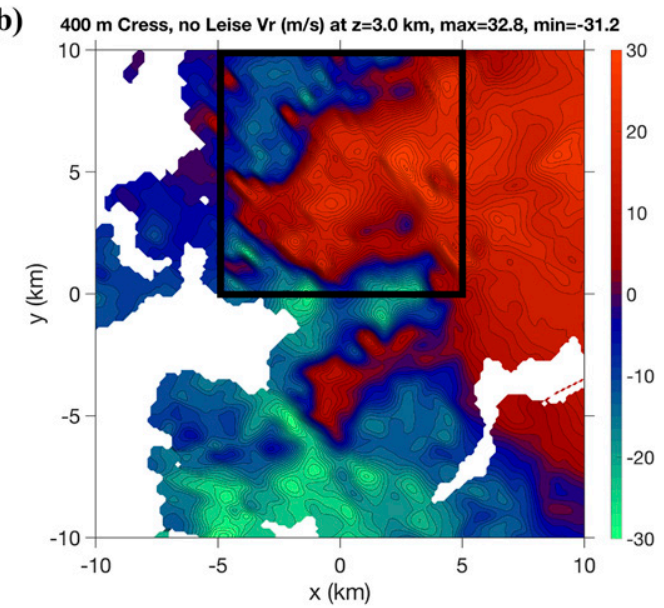

(d) $400 \mathrm{~m}$ Cress, two-pass Leise $\operatorname{Vr}(\mathrm{m} / \mathrm{s})$ at $\mathrm{z}=3.0 \mathrm{~km}$, $\max =31.9$, $\min =-31.0$

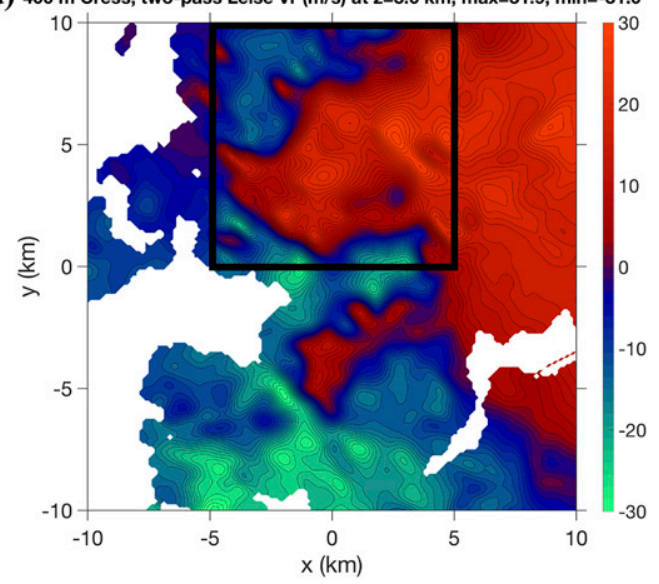

FIG. A1. Radial velocity $V_{r}$ fields at $z=3 \mathrm{~km}$ relative to the E15 R2 radar placement, interpolated to the E15 analysis grid at the analysis time. (a) "True" $V_{r}$ from the ARPS simulation. (b) Radar-observed $V_{r}$ interpolated using a 400-m Cressman radius and no Leise filter. (c) Radar-observed $V_{r}$ interpolated using an 800-m Cressman radius and no Leise filter. (d) Radar-observed $V_{r}$ interpolated using a 400-m Cressman radius and two-pass Leise filter.

$\mathrm{E} 30\left(4 \Delta=1000 \mathrm{~m}, D_{\max }=901 \mathrm{~m}\right)$, and $\mathrm{E} 45(4 \Delta=1500 \mathrm{~m}$, $\left.D_{\max }=1128 \mathrm{~m}\right)$ placements. Also, $8 \Delta$ wavelengths, which are on the order of $2 D_{\max }$, are substantially damped.

Three Cressman interpolation methods are considered here: 1 ) use a small Cressman radius (close to but greater than $0.5 D_{\max }$ to ensure that at least one observation is included in the analysis at any location) in an effort to retain as much detail as possible; 2) use a Cressman radius twice as large as that of method 1 to obtain a smoother analysis; or 3) use the same Cressman radius as in method 1 and subsequently apply a two-pass Leise filter in an effort to obtain a smooth analysis that retains detail. Each method is performed on each set of $V_{r}$ observations described in the main text. The selected Cressman radius for methods 1 and 3 is $400 \mathrm{~m}$ for the E15 placement, $500 \mathrm{~m}$ for E30, and $600 \mathrm{~m}$ for E45, while the selected Cressman radius for method 2 is $800 \mathrm{~m}$ for $\mathrm{E} 15,1000 \mathrm{~m}$ for $\mathrm{E} 30$, and $1200 \mathrm{~m}$ for $\mathrm{E} 45$. The model output $u, v, w$, and reflectivity fields at the analysis time are smoothed to the analysis grids using a Cressman radius of $\Delta$ to provide the true state corresponding to each analysis.

Visual inspection of radial velocity plots (e.g., see Fig. A1) reveals that the artifacts produced by method 1 include azimuthal discontinuities that become more apparent as the range increases (e.g., in the region plotted in Fig. A2). These artifacts stem from the use of a constant Cressman radius. They are not evident in the results from methods 2 and 3, which are compared statistically by calculating the RMS differences in $V_{r}$ and azimuthal $V_{r}$ gradient $\left[(1 / r)\left(\partial V_{r} / \partial \theta\right)\right]$ between each analysis and the true fields. The results are listed in Table A1 (for E15), Table A2 (for E30), and Table A3 (for E45). It is clear that method 3 (using the smaller Cressman radius with subsequent Leise filtering) generally 
(a)

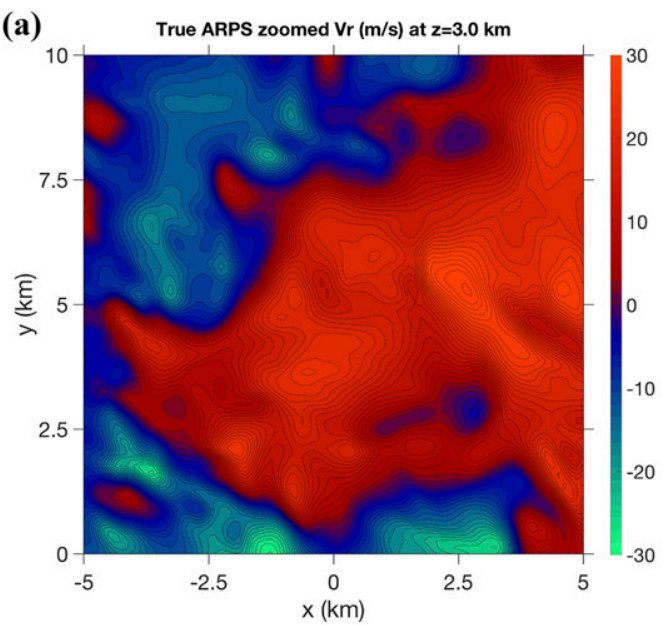

(c) $800 \mathrm{~m}$ Cress, no Leise zoomed $\operatorname{Vr}(\mathrm{m} / \mathrm{s})$ at $\mathrm{z}=3.0 \mathrm{~km}$

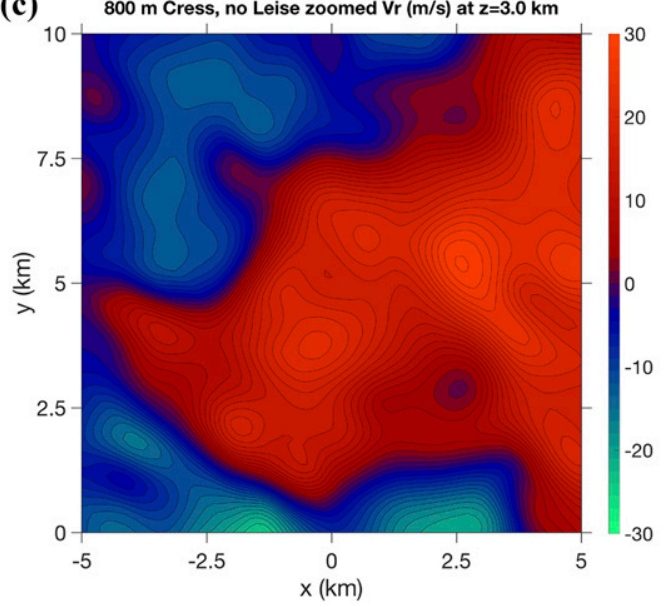



(d) $400 \mathrm{~m}$ Cress, two-pass Leise zoomed $\operatorname{Vr}(\mathrm{m} / \mathrm{s})$ at $\mathrm{z}=3.0 \mathrm{~km}$

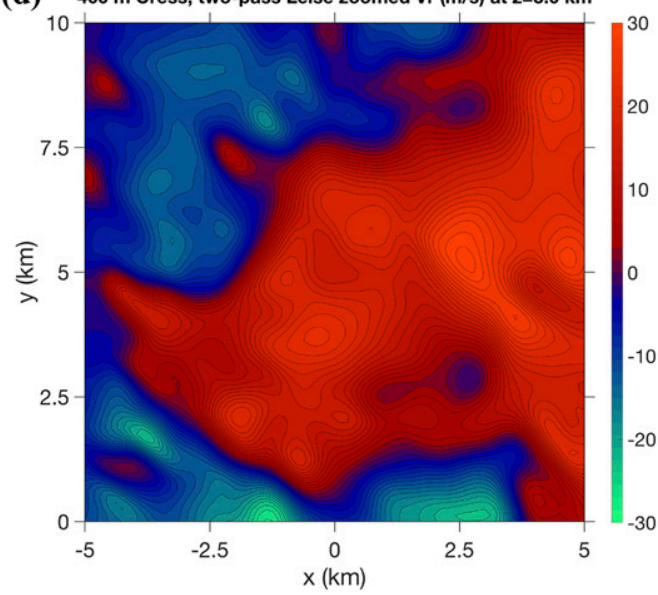

FIG. A2. As in Fig. A1, but zoomed to the regions bounded by the black boxes in that figure.

gives better statistical results than method 2 (using the larger Cressman radius). The sole exception is that the azimuthal $V_{r}$ gradient from method 2 is slightly more accurate for E45; however, in that case, the difference between the two methods is less than $10 \%$, whereas method 3 outperforms method 2 by much greater margins at closer ranges because of greater retention of finescale features. Therefore, method 3 is used for all experiments in this study.

\section{APPENDIX B}

\section{Evaluating Errors in Estimating Local Gradient Tendency for a Propagating Wave}

The task is to estimate the local tendency of the gradient in a wave $v(x, t)$ with amplitude $V$ and wavelength $L$ propagating in the $x$ direction at a speed $U$ and observed perfectly at a spatial interval $\Delta x_{o}$ and a time interval $\tau$. The amplitude varies in time as

$$
V(t)=V_{o} e^{r t},
$$

where $r$ is the rate of growth or decay. The equation for the true $v$ is

$$
v(x, t)=V_{o} e^{r t} \cos \left[\frac{2 \pi}{L}(x-U t)\right] .
$$

TABLE A1. Observation and interpolation errors in radial velocity and its azimuthal gradient for radar placement E15. Bold

\begin{tabular}{|c|c|c|c|}
\hline Radar & Analysis Method & $\begin{array}{l}V_{r} \text { RMSE } \\
\left(\mathrm{m} \mathrm{s}^{-1}\right)\end{array}$ & $\begin{array}{c}\frac{1}{r} \frac{\partial V_{r}}{\partial \theta} \mathrm{RMSE} \\
\left(10^{-3} \mathrm{~s}^{-1}\right)\end{array}$ \\
\hline \multirow[t]{2}{*}{$\mathrm{R} 1$} & $\begin{array}{l}\text { 800-m Cressman, } \\
\text { no Leise }\end{array}$ & 2.36 & 9.28 \\
\hline & $\begin{array}{l}\text { 400-m Cressman, } \\
\text { two-pass Leise }\end{array}$ & 1.74 & 7.32 \\
\hline \multirow[t]{2}{*}{$\mathrm{R} 2$} & $\begin{array}{l}\text { 800-m Cressman, } \\
\text { no Leise }\end{array}$ & 2.18 & 8.55 \\
\hline & $\begin{array}{l}\text { 400-m Cressman, } \\
\text { two-pass Leise }\end{array}$ & 1.56 & 6.81 \\
\hline
\end{tabular}
font highlights best performance for each radar. 
TABLE A2. As in Table A1, but for radar placement E30.

\begin{tabular}{cccc}
\hline Radar & Analysis Method & $\begin{array}{c}V_{r} \text { RMSE } \\
\left(\mathrm{m} \mathrm{s}^{-1}\right)\end{array}$ & $\begin{array}{c}\frac{1}{r} \frac{\partial V_{r}}{\partial \theta} \mathrm{RMSE} \\
\left(10^{-3} \mathrm{~s}^{-1}\right)\end{array}$ \\
\hline $\mathrm{R} 1$ & $\begin{array}{c}\text { 1000-m Cressman, } \\
\text { no Leise }\end{array}$ & 2.45 & 6.62 \\
& $\begin{array}{c}\text { 500-m Cressman, } \\
\text { two-pass Leise } \\
\text { R2 }\end{array}$ & $\mathbf{2 . 0 7}$ & $\mathbf{6 . 1 1}$ \\
& $\begin{array}{c}\text { 1000-m Cressman, } \\
\text { no Leise } \\
\text { 500-m Cressman, } \\
\text { two-pass Leise }\end{array}$ & 2.40 & 6.22 \\
& $\mathbf{2 . 0 7}$ & $\mathbf{5 . 7 9}$ \\
\hline
\end{tabular}

All errors are calculated relative to the truth filtered to the analysis grid using Cressman interpolation with a cutoff radius $r_{a}$ set to the analysis grid spacing. The filtered truth $v_{f}$ is given by

$$
v_{f}(x, t)=\frac{\int_{x-r_{a}}^{x+r_{a}}\left[\frac{r_{a}^{2}-\left(x^{\prime}-x\right)^{2}}{r_{a}^{2}+\left(x^{\prime}-x\right)^{2}}\right] v\left(x^{\prime}, t\right) d x^{\prime}}{\int_{x-r_{a}}^{x+r_{a}}\left[\frac{r_{a}^{2}-\left(x^{\prime}-x\right)^{2}}{r_{a}^{2}+\left(x^{\prime}-x\right)^{2}}\right] d x^{\prime}} .
$$

Applying (B3) in (B2) and substituting $\gamma \equiv x^{\prime}-x$ yields

$$
v_{f}(x, t)=V_{f} e^{r t} \cos \left[\frac{2 \pi}{L}(x-U t)\right],
$$

where

$$
V_{f}=V_{o} \frac{\int_{-r_{a}}^{r_{a}}\left(\frac{r_{a}^{2}-\gamma^{2}}{r_{a}^{2}+\gamma^{2}}\right) \cos \left(\frac{2 \pi \gamma}{L}\right) d \gamma}{\int_{-r_{a}}^{r_{a}}\left(\frac{r_{a}^{2}-\gamma^{2}}{r_{a}^{2}+\gamma^{2}}\right) d \gamma} .
$$

The true value of the filtered local gradient tendency is given by

$$
\begin{aligned}
\frac{\partial^{2} v_{f}}{\partial x \partial t}(x, t)= & \left(\frac{2 \pi}{L}\right)^{2} U V_{f} e^{r t} \cos \left[\frac{2 \pi}{L}(x-U t)\right] \\
& -\frac{2 \pi}{L} r V_{f} e^{r t} \sin \left[\frac{2 \pi}{L}(x-U t)\right] .
\end{aligned}
$$

\begin{tabular}{|c|c|c|c|}
\hline Radar & Analysis Method & $\begin{array}{l}V_{r} \text { RMSE } \\
\left(\mathrm{m} \mathrm{s}^{-1}\right)\end{array}$ & $\begin{array}{c}\frac{1}{r} \frac{\partial V_{r}}{\partial \theta} \mathrm{RMSE} \\
\left(10^{-3} \mathrm{~s}^{-1}\right)\end{array}$ \\
\hline \multirow[t]{2}{*}{$\mathrm{R} 1$} & $\begin{array}{l}\text { 1200-m Cressman, } \\
\text { no Leise }\end{array}$ & 2.66 & 5.07 \\
\hline & $\begin{array}{l}\text { 600-m Cressman, } \\
\text { two-pass Leise }\end{array}$ & 2.59 & 5.37 \\
\hline \multirow[t]{2}{*}{$\mathrm{R} 2$} & $\begin{array}{l}\text { 1200-m Cressman, } \\
\text { no Leise }\end{array}$ & 2.64 & 4.65 \\
\hline & $\begin{array}{c}\text { 600-m Cressman, } \\
\text { two-pass Leise }\end{array}$ & 2.61 & 4.93 \\
\hline
\end{tabular}

For an estimate valid at time $t$, observations of the true, unfiltered $v$ given by (B2) are taken at times $t-\tau$ and $t+\tau$ at locations $m \Delta x_{o}$, where $m$ is an integer. Cressman
TABLE A3. As in Table A1, but for radar placement E45.

interpolation with cutoff radius $r_{c}$ is used to map these observations to a stencil of 11 analysis grid points centered at $x$ with spacing $\Delta x_{a}$. A two-pass Leise filter is applied to the stencil to obtain intermediate values $v_{\text {int }}$ at locations $x-\Delta x_{a}$ and $x+\Delta x_{a}$. Random, unbiased error is then added to $v_{\text {int }}$ to approximate the error introduced by the provisional retrievals. For a given radar placement, the standard deviation of this error is set equal to the root-meansquare error for the corresponding provisional retrievals of $v\left(v^{p}\right.$ RMSE), calculated with respect to the filtered truth $v_{f}$. Thus, the final analyzed value of $v$ is given by

$$
v^{a}(x, t)=v_{\mathrm{int}}(x, t)+N\left(0, v^{p} \mathrm{RMSE}\right),
$$

where $N\left(0, v^{p}\right.$ RMSE) denotes a normally distributed random value with mean 0 and standard deviation equal to the $v^{p}$ RMSE.

Centered differences are used to estimate the local tendency of the gradient at $x$ :

$$
\begin{aligned}
\left.\frac{\delta}{\delta t}\left(\frac{\delta v^{a}}{\delta x}\right)\right|_{x, t}= & \frac{1}{4 \Delta x_{a} \tau}\left[v^{a}\left(x+\Delta x_{a}, t+\tau\right)\right. \\
& -v^{a}\left(x-\Delta x_{a}, t+\tau\right)-v^{a}\left(x+\Delta x_{a}, t-\tau\right) \\
& \left.+v^{a}\left(x-\Delta x_{a}, t-\tau\right)\right] .
\end{aligned}
$$

The total interpolation error at $\left(x, t^{a}\right)$ is

$$
\begin{gathered}
E\left(V_{o}, x, \Delta x_{o}, \Delta x_{a}, r_{o}, t^{a}, \tau, L, U, r\right) \\
\quad=\frac{\partial^{2} v_{f}}{\partial x \partial t}\left(x, t^{a}\right)-\left.\frac{\delta}{\delta t}\left(\frac{\delta v^{a}}{\delta x}\right)\right|_{x, t^{a}} .
\end{gathered}
$$

To simplify matters, the analysis time as set to $t^{a}=0$. The error is also normalized by $(2 \pi / L)^{2} U V_{f}$ [the maximum value of (B6) when $r=0$ and $t=0$ ], which gives

$$
\begin{aligned}
E\left(x, \Delta x_{o}, \Delta x_{a}, r_{o}, \tau, L, U, r\right)= & \cos \left(\frac{2 \pi x}{L}\right)-\frac{r L}{2 \pi U} \sin \left(\frac{2 \pi x}{L}\right)-\frac{1}{4 \Delta x_{a} \tau U V_{f}}\left(\frac{L}{2 \pi}\right)^{2}\left[v^{a}\left(x+\Delta x_{a}, \tau\right)\right. \\
& \left.-v^{a}\left(x-\Delta x_{a}, \tau\right)-v^{a}\left(x+\Delta x_{a},-\tau\right)+v^{a}\left(x-\Delta x_{a},-\tau\right)\right] .
\end{aligned}
$$


Assuming the observation spacing $\Delta x_{o}$ and the wavelength $L$ are both integer multiples of a common length scale $\Delta x_{s}$ (e.g., $\Delta x_{s}=1 \mathrm{~m}$ if $\Delta x_{o}$ and $L$ are both expressed to the nearest meter), the interpolation error is periodic in $x$ with a period of (at most) $L_{s}=\kappa L$, where $\kappa=\Delta x_{o} / \Delta x_{s}$. The global RMSE is then given by

$$
\begin{aligned}
& \operatorname{RMSE}\left(\Delta x_{o}, \Delta x_{a}, r_{o}, \tau, L, U, r\right) \\
& =\left\{\frac{1}{L_{s}} \int_{0}^{L_{s}}\left[E\left(x, \Delta x_{o}, \Delta x_{a}, r_{o}, \tau, L, U, r\right)\right]^{2} d x\right\}^{0.5} .
\end{aligned}
$$

An analytic solution for (B11) is difficult to obtain because of the interpolation and filtering used to obtain $v^{a}$. Instead, the integral is treated as a discrete sum over $K\left(=L_{s} / \Delta x_{s}\right)$ points, providing the normalized global RMSE values reported in section $3 \mathrm{~b}$ :

$$
\begin{aligned}
& \operatorname{RMSE}\left(\Delta x_{o}, \Delta x_{a}, r_{o}, \tau, L, U, r\right) \\
& =\left\{\left(\frac{1}{K+1}\right) \sum_{k=0}^{K}\left[E\left(k \Delta x_{s}, \Delta x_{o}, \Delta x_{a}, r_{o}, \tau, L, U, r\right)\right]^{2}\right\}^{0.5} .
\end{aligned}
$$

\section{REFERENCES}

Armijo, L., 1969: A theory for the determination of wind and precipitation velocities with Doppler radars. J. Atmos. Sci., 26, 570-573, https://doi.org/10.1175/1520-0469(1969)026<0570: ATFTDO $>2.0 . \mathrm{CO} ; 2$.

Atkins, N. T., E. M. Glidden, and T. M. Nicholson, 2014: Observations of wall cloud formation in supercell thunderstorms during VORTEX2. Mon. Wea. Rev., 142, 4823-4838, https:// doi.org/10.1175/MWR-D-14-00125.1.

Atlas, D., R. Srivastava, and R. Sekhon, 1973: Doppler radar characteristics of precipitation at vertical incidence. Rev. Geophys. Space Phys., 11, 1-35, https://doi.org/10.1029/RG011i001p00001.

Beck, J. R., J. L. Schroeder, and J. M. Wurman, 2006: Highresolution dual-Doppler analyses of the 29 May 2001 Kress, Texas, cyclic supercell. Mon. Wea. Rev., 134, 3125-3148, https://doi.org/10.1175/MWR3246.1.

Brandes, E. A., 1977: Flow in severe thunderstorms observed by dualDoppler radar. Mon. Wea. Rev., 105, 113-120, https://doi.org/ 10.1175/1520-0493(1977)105<0113:FISTOB >2.0.CO;2.

$\ldots$, 1984a: Relationship between radar-derived thermodynamic variables and tornadogenesis. Mon. Wea. Rev., 112, 1033-1052, https:// doi.org/10.1175/1520-0493(1984)112<1033:RBRDTV>2.0.CO;2.

,$- 1984 \mathrm{~b}$ : Vertical vorticity generation and mesocyclonic sustenance in tornadic thunderstorms: The observational evidence. Mon. Wea. Rev., 112, 2253-2269, https://doi.org/ 10.1175/1520-0493(1984)112<2253:VVGAMS > 2.0.CO;2.

Byun, D. W., 1990: On the analytical solutions of the flux-profile relationships for the atmospheric surface layer. J. Appl. Meteor., 29, 652-657, https://doi.org/10.1175/1520-0450(1990) $029<0652$ :OTASOF $>2.0$. CO 2 .

Cai, H., 2005: Comparison between tornadic and nontornadic mesocyclones using the vorticity (pseudovorticity) line technique. Mon. Wea. Rev., 133, 2535-2551, https://doi.org/10.1175/ MWR2990.1.

Calhoun, K. M., D. R. MacGorman, C. L. Ziegler, and M. I. Biggerstaff, 2013: Evolution of lightning activity and storm charge relative to dual-Doppler analysis of a high-precipitation supercell storm. Mon. Wea. Rev., 141, 2199-2223, https://doi.org/ 10.1175/MWR-D-12-00258.1.

Carbone, R. E., 1982: A severe frontal rainband. Part I: Stormwide hydrodynamic structure. J. Atmos. Sci., 39, 258-279, https:// doi.org/10.1175/1520-0469(1982)039<0258:ASFRPI>2.0.CO;2.

Chong, M., and J. Testud, 1983: Three-dimensional wind field analysis from dual-Doppler radar data. Part III: The boundary condition: An optimum determination based on a variational concept. J. Climate Appl. Meteor., 22, 1227-1241, https://doi.org/10.1175/ 1520-0450(1983)022<1227:TDWFAF $>2.0 . C O ; 2$.

Clark, T. L., F. I. Harris, and C. G. Mohr, 1980: Errors in wind fields derived from multiple-Doppler radars: Random errors and temporal errors associated with advection and evolution. J. Appl. Meteor., 19, 1273-1284, https://doi.org/10.1175/15200450(1980)019<1273:EIWFDF>2.0.CO;2.

Cressman, G. P., 1959: An operational objective analysis system. Mon. Wea. Rev., 87, 367-374, https://doi.org/10.1175/15200493(1959)087<0367:AOOAS>2.0.CO;2.

Dahl, N. A., and D. S. Nolan, 2018: Using high-resolution simulations to quantify errors in radar estimates of tornado intensity. Mon. Wea. Rev., 146, 2271-2296, https://doi.org/10.1175/ MWR-D-17-0333.1.

Doviak, R. J., and D. S. Zrnić, 1993: Doppler Radar and Weather Observations. Academic Press, 562 pp.

Dowell, D. C., and H. B. Bluestein, 1997: The Arcadia, Oklahoma, storm of 17 May 1981: Analysis of a supercell during tornadogenesis. Mon. Wea. Rev., 125, 2562-2582, https://doi.org/ 10.1175/1520-0493(1997)125<2562:TAOSOM>2.0.CO;2.

— , and —_, 2002a: The 8 June 1995 McLean, Texas, storm. Part I: Observations of cyclic tornadogenesis. Mon. Wea. Rev., 130, 2626-2648, https://doi.org/10.1175/1520-0493(2002)130<2626: TJMTSP $>2.0 . C O ; 2$.

— Part II: Cyclic tornado formation, maintenance, and dissipation. Mon. Wea. Rev., 130, 2649-2670, https://doi.org/10.1175/ 1520-0493(2002)130<2649:TJMTSP > 2.0.CO;2.

_ synthesis in Cartesian coordinates. J. Atmos. Oceanic Technol., 20, 1552-1559, https://doi.org/10.1175/1520-0426(2003)020<1552: SOAIDW $>2.0 . \mathrm{CO} ; 2$.

Falcone, M., and R. Ferretti, 1998: Convergence analysis for a class of high-order semi-Lagrangian advection schemes. SIAM J. Numer. Anal., 35, 909-940, https://doi.org/10.1137/S0036142994273513.

Ferrell, W., and Coauthors, 2014: Atmospheric Research Measurement Climate Research Facility-Atmospheric System Research high-resolution modeling workshop. Office of Science Rep. DOE/SC-0169, 46 pp., https://science.osti.gov/ /media/ber/pdf/ workshop\%20reports/doe-sc-0169-low-resolution.pdf.

Frame, J., P. Markowski, Y. Richardson, J. Straka, and J. Wurman, 2009: Polarimetric and dual-Doppler radar observations of the Lipscomb County, Texas, supercell thunderstorm on 23 May 2002. Mon. Wea. Rev., 137, 544-564, https://doi.org/10.1175/ 2008MWR2425.1.

Gal-Chen, T., and R. A. Kropfli, 1984: Buoyancy and pressure perturbations derived from dual-Doppler radar observations of the planetary boundary layer: Applications for matching models with observations. J. Atmos. Sci., 41, 3007-3020, https://doi.org/ 10.1175/1520-0469(1984)041<3007:BAPPDF>2.0.CO;2. 
Gao, J., M. Xue, A. Shapiro, and K. K. Droegemeier, 1999: A variational method for the analysis of three-dimensional wind fields from two Doppler radars. Mon. Wea. Rev., 127, 2128-2142, https:// doi.org/10.1175/1520-0493(1999)127<2128:AVMFTA>2.0.CO;2.

, K. Brewster, and K. K. Droegemeier, 2004: A threedimensional variational data analysis method with recursive filter for Doppler radars. J. Atmos. Oceanic Technol., 21, 457-469, https://doi.org/10.1175/1520-0426(2004)021<0457: ATVDAM $>2.0 . \mathrm{CO} ; 2$.

Isom, B., and Coauthors, 2013: The Atmospheric Imaging Rader: Simultaneous volumetric observations using a phased array weather radar. J. Atmos. Oceanic Technol., 30, 655-675, https://doi.org/10.1175/JTECH-D-12-00063.1.

Kessinger, C. J., P. S. Ray, and C. E. Hane, 1987: The Oklahoma squall line of 19 May 1977. Part I: A multiple Doppler analysis of convective and stratiform structure. J. Atmos. Sci., 44, 2840-2864, https://doi.org/10.1175/1520-0469(1987)044<2840: TOSLOM $>2.0 . \mathrm{CO} ; 2$.

Kosiba, K., and J. Wurman, 2014: Finescale dual-Doppler analysis of hurricane boundary layer structures in Hurricane Frances (2004) at landfall. Mon. Wea. Rev., 142, 1874-1891, https:// doi.org/10.1175/MWR-D-13-00178.1.

__, _- , Y. Richardson, P. Markowski, P. Robinson, and J. Marquis, 2013: Genesis of the Goshen County, Wyoming, tornado on 5 June 2009 during VORTEX2. Mon. Wea. Rev., 141, 1157-1181, https://doi.org/10.1175/MWR-D-1200056.1

Lee, W.-C., R. E. Carbone, and R. M. Wakimoto, 1992: The evolution and structure of a "bow-echo microburst" event. Part I: The microburst. Mon. Wea. Rev., 120, 2188-2210, https://doi.org/ 10.1175/1520-0493(1992)120<2188:TEASOA > 2.0.CO;2.

Leise, J. A., 1982: A multidimensional scale-telescoped filter and data extension package. NOAA Tech. Memo. ERL WPL-82, 19 pp.

Markowski, P., and Coauthors, 2012: The pretornadic phase of the Goshen County, Wyoming, supercell of 5 June 2009 intercepted by VORTEX2. Part I: Evolution of kinematic and surface thermodynamic fields. Mon. Wea. Rev., 140, 2887 2915, https://doi.org/10.1175/MWR-D-11-00336.1.

_, T. Hatlee, and Y. Richardson, 2018: Tornadogenesis in the 12 May 2010 supercell thunderstorm intercepted by VORTEX2 near Clinton, Oklahoma. Mon. Wea. Rev., 146, 3623-3650, https://doi.org/10.1175/MWR-D-18-0196.1.

Marquis, J., Y. Richardson, J. Wurman, and P. Markowski, 2008: Single- and dual-Doppler analysis of a tornadic vortex and surrounding storm-scale flow in the Crowell, Texas, supercell of 30 April 2000. Mon. Wea. Rev., 136, 5017-5043, https:// doi.org/10.1175/2008MWR2442.1.

— - _ P. Markowski, D. Dowell, and J. Wurman, 2012: Tornado maintenance investigated with high-resolution dual-Doppler and EnKF analysis. Mon. Wea. Rev., 140, 3-27, https://doi.org/10.1175/MWR-D-11-00025.1.

Mewes, J. J., and A. Shapiro, 2002: On the use of the vorticity equation in dual-Doppler analysis of the vertical velocity field. J. Atmos. Oceanic Technol., 19, 543-567, https://doi.org/ 10.1175/1520-0426(2002)019<0543:UOTVEI >2.0.CO;2.

Parsons, D. B., and R. A. Kropfli, 1990: Dynamics and fine structure of a microburst. J. Atmos. Sci., 47, 1674-1692, https://doi.org/ 10.1175/1520-0469(1990)047<1674:DAFSOA > 2.0.CO;2.

Potvin, C. K., A. Shapiro, T.-Y. Yu, J. Gao, and M. Xue, 2009: Using a low-order model to detect and characterize tornadoes in multi-Doppler radar data. Mon. Wea. Rev., 137, 1230-1249, https://doi.org/10.1175/2008MWR2446.1.
_ D. Betten, L. J. Wicker, K. L. Elmore, and M. I. Biggerstaff, 2012a: 3DVAR versus traditional dual-Doppler wind retrievals of a simulated supercell thunderstorm. Mon. Wea. Rev., 140, 3487-3494, https://doi.org/10.1175/MWR-D-12-00063.1.

_ A. Shapiro, and M. Xue, 2012b: Impact of a vertical vorticity constraint in variational dual-Doppler wind analysis: Tests with real and simulated supercell data. J. Atmos. Oceanic Technol., 29, 32-49, https://doi.org/10.1175/JTECH-D-11-00019.1.

Press, W. H., S. A. Teukolsky, W. T. Vetterling, and B. P. Flannery, 1992: Numerical Recipes in Fortran: The Art of Scientific Computing. 2nd ed. Cambridge University Press, 963 pp.

Ray, P. S., R. J. Doviak, G. B. Walker, D. Sirmans, J. Carter, and B. Baumgarner, 1975: Dual-Doppler observations of a tornadic storm. J. Appl. Meteor., 14, 1521-1530, https://doi.org/ 10.1175/1520-0450(1975)014<1521:DDOOAT >2.0.CO;2.

— C. L. Ziegler, W. Bumgarner, and R. J. Serafin, 1980: Singleand multiple-Doppler radar observations of tornadic storms. Mon. Wea. Rev., 108, 1607-1625, https://doi.org/10.1175/15200493(1980)108<1607:SAMDRO > 2.0.CO;2.

_ D. P. Jorgensen, and S.-L. Wang, 1985: Airborne Doppler radar observations of a convective storm. J. Climate Appl. Meteor., 24, 687-698, https://doi.org/10.1175/1520-0450(1985) 024<0687:ADROOA > 2.0.CO;2.

Roux, F., J. Testud, M. Payen, and B. Pinty, 1984: West African squall line thermodynamic structure retrieved from dual-Doppler radar observations. J. Atmos. Sci., 41, 3104-3121, https://doi.org/ 10.1175/1520-0469(1984)041<3104:WASLTS>2.0.CO;2.

Schenkman, A. D., M. Xue, and M. Hu, 2014: Tornadogenesis in a high-resolution simulation of the 8 May 2003 Oklahoma City supercell. J. Atmos. Sci., 71, 130-154, https://doi.org/10.1175/ JAS-D-13-073.1.

Shao, A., C. Qiu, and L. Liu, 2004: Kinematic structure of a heavy rain event from dual-Doppler radar observations. Adv. Atmos. Sci., 21, 609-616, https://doi.org/10.1007/BF02915728.

Shapiro, A., C. K. Potvin, and J. Gao, 2009: Use of a vertical vorticity equation in variational dual-Doppler wind analysis. J. Atmos. Oceanic Technol., 26, 2089-2106, https://doi.org/ 10.1175/2009JTECHA1256.1.

_, K. M. Willingham, and C. K. Potvin, 2010a: Spatially variable advection correction of radar data. Part I: Theoretical considerations. J. Atmos. Sci., 67, 3445-3456, https://doi.org/ 10.1175/2010JAS3465.1.

,-- , and,$- 2010 \mathrm{~b}$ : Spatially variable advection correction of radar data. Part II: Test results. J. Atmos. Sci., 67, 3457-3470, https://doi.org/10.1175/2010JAS3466.1.

Snyder, J. C., and H. B. Bluestein, 2014: Some considerations for the use of mobile Doppler radar data for tornado intensity determination. Wea. Forecasting, 29, 799-827, https://doi.org/ 10.1175/WAF-D-14-00026.1.

Trapp, R. J., and C. A. Doswell, 2000: Radar data objective analysis. J. Atmos. Oceanic Technol., 17, 105-120, https://doi.org/ 10.1175/1520-0426(2000)017<0105:RDOA > 2.0.CO;2.

Wienhoff, Z. B., H. B. Bluestein, L. J. Wicker, J. C. Snyder, A. Shapiro, C. K. Potvin, J. B. Houser, and D. W. Reif, 2018: Applications of a spatially variable advection correction technique for temporal correction of dual-Doppler analyses of tornadic supercells. Mon. Wea. Rev., 146, 2949-2971, https:// doi.org/10.1175/MWR-D-17-0360.1.

Wingo, S. M., and K. R. Knupp, 2016: Kinematic structure of mesovortices in the eyewall of Hurricane Ike (2008) derived from ground-based dual-Doppler analysis. Mon. Wea. Rev., 144, 4245-4263, https://doi.org/10.1175/MWR-D-160085.1. 
Wurman, J., Y. Richardson, C. Alexander, S. Weygandt, and P. F. Zhang, 2007: Dual-Doppler analysis of winds and vorticity budget terms near a tornado. Mon. Wea. Rev., 135, 2392-2405, https://doi.org/10.1175/MWR3404.1.

Xue, M., K. K. Droegemeier, and V. Wong, 2000: The Advanced Regional Prediction System (ARPS)-A multiscale nonhydrostatic atmospheric simulation and prediction tool. Part I: Model dynamics and verification. Meteor. Atmos. Phys., 75, 161-193, https://doi.org/10.1007/s007030070003.

, and Coauthors, 2001: The Advanced Regional Prediction System (ARPS)—A multiscale non-hydrostatic atmospheric simulation and prediction tool. Part II: Model physics and applications. Meteor. Atmos. Phys., 76, 143-165, https:// doi.org/10.1007/s007030170027.

- M. Hu, and A. Schenkman, 2014: Numerical prediction of 8 May 2003 Oklahoma City tornadic supercell and embedded tornado using ARPS with the assimilation of WSR-88D data. Wea. Forecasting, 29, 39-62, https://doi.org/10.1175/WAF-D13-00029.1.

Ziegler, C., P. S. Ray, and N. C. Knight, 1983: Hail growth in an Oklahoma multicell storm. J. Atmos. Sci., 40, 1768-1791, https:// doi.org/10.1175/1520-0469(1983)040<1768:HGIAOM>2.0.CO;2. 